\title{
Maize Grain Extract Enriched with Polyamines Alleviates Drought Stress in Triticum aestivum through Up-Regulation of the Ascorbate-Glutathione Cycle, Glyoxalase System, and Polyamine Gene Expression
}

\author{
Hesham F. Alharby ${ }^{1}$, Hassan S. Al-Zahrani ${ }^{1}$, Yahya M. Alzahrani ${ }^{1}$, Hameed Alsamadany ${ }^{1}$ (D), \\ Khalid R. Hakeem ${ }^{1, *(D)}$ and Mostafa M. Rady ${ }^{2} \mathbb{D}$ \\ 1 Department of Biological Sciences, Faculty of Science, King Abdulaziz University, \\ Jeddah 21589, Saudi Arabia; halharby@kau.edu.sa (H.F.A.); hsalzahrani@kau.edu.sa (H.S.A.-Z.); \\ yalzahrani@kau.edu.sa (Y.M.A.); halsamadani@kau.edu.sa (H.A.) \\ 2 Botany Department, Faculty of Agriculture, Fayoum University, Fayoum 63514, Egypt; \\ mmr02@fayoum.edu.eg or mrady2050@gmail.com \\ * Correspondence: kur.hakeem@gmail.com or khakim@kau.edu.sa; Tel.: +966-508-642-544
}

\section{check for} updates

Citation: Alharby, H.F.; Al-Zahrani, H.S.; Alzahrani, Y.M.; Alsamadany,

H.; Hakeem, K.R.; Rady, M.M.

Maize Grain Extract Enriched with

Polyamines Alleviates Drought

Stress in Triticum aestivum

through Up-Regulation of the

Ascorbate-Glutathione Cycle,

Glyoxalase System, and Polyamine

Gene Expression. Agronomy 2021, 11, 949. https://doi.org/10.3390/

agronomy11050949

Received: 8 April 2021

Accepted: 6 May 2021

Published: 11 May 2021

Publisher's Note: MDPI stays neutral with regard to jurisdictional claims in published maps and institutional affiliations.

Copyright: (c) 2021 by the authors. Licensee MDPI, Basel, Switzerland. This article is an open access article distributed under the terms and conditions of the Creative Commons Attribution (CC BY) license (https:// creativecommons.org/licenses/by/ $4.0 /)$.

\begin{abstract}
The potential protective role of priming wheat seeds with maize green extract (MGE) against the stress effects of drought was studied. Pretreatment using MGE, MGE enriched with polyamines (MGE $\mathrm{PA}_{\mathrm{PA}}$ ), and drought treatments (irrigation deficit of 30\% (severe drought) or $60 \%$ (moderate drought) versus $90 \%$ relative water content of soil as a control) were applied in a factorial completely randomized design. Under moderate drought, pretreatment with $\mathrm{MGE}_{\mathrm{PA}}$ outperformed MGE and control, while severely stressed plants died even with pretreatments. Both extracts enhanced normal plant growth and yield and mitigated the deleterious effect of moderately stressed plants. Application of both extracts markedly increased photosynthetic efficiency, membrane stability, relative water content, and accumulation of antioxidants, osmoprotectants, trans- and cis-zeatin, polyamines, and their gene expressions, while levels of superoxide $\left(\mathrm{O}_{2}{ }^{\bullet-}\right)$ and hydrogen peroxide $\left(\mathrm{H}_{2} \mathrm{O}_{2}\right)$, lipid peroxidation, and electrolyte leakage were decreased. Enzymatic antioxidants and glyoxalase system activities were improved in moderately stressed plants and were further improved with pretreatment with both extracts, thus protecting plants from oxidative damage by up-regulation of the ascorbate-glutathione cycle. Glycine betaine, soluble sugars, and proline levels were greatly increased in pretreated plants, thus maintaining membrane stability and photosynthetic efficiency. The interaction between drought and pretreatment using $\mathrm{MGE}_{\mathrm{PA}}$ was significant in growing wheat plants in dry environments with $60 \%$ relative water content of soil.
\end{abstract}

Keywords: wheat; performance; antioxidant system; ascorbate-glutathione cycle; glyoxalase system; gene expressions; plant water status

\section{Introduction}

Drought, one of the major types of environmental abiotic stresses, restricts plant performance throughout its life cycle. It limits the productivity of agricultural crops, mostly in dry areas (e.g., semi-arid and arid regions), including Egypt and Saudi Arabia, thus causing an increasing problem over time due to the ongoing climate change. Deficit irrigation water (DIw) adversely affects plant growth, development, and production through decreasing water flux, closure of stomata, and reduced $\mathrm{CO}_{2}$ fixation [1-3]. DIw strongly affects plant antioxidant system, metabolism, and physio-biochemistry [4-8]. It causes photooxidation damage to cell components by over-generating reactive species of oxygen (ROS), including radicals of $\mathrm{O}_{2}{ }^{\bullet-}$ and $\mathrm{H}_{2} \mathrm{O}_{2}$ and eventually photo-inhibition [9], which cause significant oxidative damage to proteins, lipids, and DNA, thus discouraging plant growth [10]. To mitigate the oxidative stress damage, complex ROS-scavenging antioxidant 
system (enzymes (SOD, POD, CAT, etc.) and low-molecular mass compounds (GSH, AsA, carotenoids, phenols, etc.)) are developed/adopted in plants [11]. The enzymatic antioxidants can directly scavenge ROS or produce one or more of the non-enzymatic antioxidants. SOD is specifically implicated in converting $\mathrm{O}_{2}{ }^{\bullet-}$ to $\mathrm{H}_{2} \mathrm{O}_{2}$ in cell cytoplasm, chloroplasts, mitochondria, and peroxisomes. In addition, POD acts as a scavenger of $\mathrm{H}_{2} \mathrm{O}_{2}$ and CAT eliminates the $\mathrm{H}_{2} \mathrm{O}_{2}$ in mitochondria and micro-bodies [12], helping mitigate the adverse effects of oxidative stress.

The effective reduction of cell osmotic potential maintains plant cell metabolism and avoids dehydration damage induced by DIw stress via the accumulation of more levels of glycinebetaine, soluble sugars, and proline [13]. These osmotic compounds maintain an osmotic balance, efficacy, and normality of membrane functions, and protect macromolecules and plasma membranes, providing cellular resistance against DIw stress and dehydration $[10,14,15]$.

In most cases, endogenous components of the developed/adopted antioxidant system in plants are not sufficient to defend against environmental stress. Consequently, plant extract and microbial bio-stimulants may be applied exogenously as an adjuvant to plants to increase their tolerance to stress. Plant extracts can induce plant resistance under various stresses, and microbial biostimulants can increase gene regulation in plants related to drought stress and can also produce biofilm, which helps plants survive under drought stress [16-21].

At present, extracts containing natural bio-stimulants obtained from maize grains have been applied for seed priming and have been reported to enhance plant performances under different stress conditions; salinity [22,23], nutrient deficiencies [24], cadmium [25], and salinity plus drought [26]. MGE is an important organic bio-stimulant for different stressed plants. It is rich in various phytohormones (e.g., cytokinins, auxins, and gibberellins), antioxidants, and essential nutrients. It has been used to enhance plant morphology, physiology, biochemistry, and induce plant tolerance against stress damages [22,25]. To date, no work has been done using MGE enriched with antioxidants such as polyamines for wheat grown under DIw stress. Thus, this is the first report that used MGE to stimulate the growth of pretreated wheat plants under DIw stress due to its high content of antioxidants, plant hormones, vitamins, polyamines, and nutrients, making it an effective biological stimulant [22,24-27] (Table 1). These reports stated that MGE confers seed and their emerged seedlings the ability to resist the unfavorable effects of stress through elevating wheat performance (growth and productivity), physio-biochemical parameters, and different antioxidants. Thus, MGE is an innovative means to provide seeds and developing seedlings that are resistant to damages caused by environmental stresses such as DIw.

Table 1. Antioxidant and hormone contents detected in maize grain extract (MGE).

\begin{tabular}{ccc}
\hline Content & Unit & Value \\
\hline & Antioxidants: & $4.26 \pm 0.07$ \\
Ascorbic acid (AsA) & $\left(\mu \mathrm{mol} \mathrm{g}^{-1} \mathrm{FW}\right)$ & $1.85 \pm 0.03$ \\
Glutathione (GSH) & $\%$ & $88.6 \pm 1.6$ \\
DPPH radical-scavenging activity & Polyamines: & $0.5 \pm 0.01$ \\
Putrescine (PUT) & $\left(\mu \mathrm{mol} \mathrm{g}^{-1} \mathrm{FW}\right)$ & $0.6 \pm 0.01$ \\
Spermidine (SPD) & & $0.9 \pm 0.02$ \\
Spermine (SPM) & Phytohormones: & $3.56 \pm 0.06$ \\
Total cytokinins (CKs) & & $1.55 \pm 0.02$ \\
Trans-Zeatin $(t-Z)$ & $\left(\mu \mathrm{mol} \mathrm{g}{ }^{-1} \mathrm{FW}\right)$ & $1.02 \pm 0.01$ \\
Cis-Zeatin $(c-Z)$ & & $2.29 \pm 0.05$ \\
Salicylic acid (SA) & & \\
\hline
\end{tabular}

Values presented in the table are means $(n=3$ for all measures $) \pm$ standard error.

Wheat (Triticum aestivum L.) grain and straw are major sources of nourishing the diets of humans and animals, respectively in urban and rural communities due to their high protein and calorie content (approximately $82-85 \%$ ). However, environmental stresses 
severely affect wheat productivity; if the available soil water reaches $45-50 \%$ of field capacity in the root zone, the wheat plants must be watered [2,26]. The duration and severity of stress, growth physio-biochemical processes, and patterns of differential gene expression are some of the factors that can influence the wheat plant's response to DIw stress $[23,26,28,29]$.

Although MGE has been used in some investigations for some crop plants to boost their antioxidant defense system against the stress impacts of certain abiotic stresses [22,24-27], there is no information available on seed priming using MGE (as a bio-stimulant) to encourage plant growth and production by mitigating the damage caused by drought stress. However, by analyzing MGE, we found that it is rich in phytohormones, especially zeatins, but was poor in PAs (Table 1), so enriching this extract with PAs could increase its effectiveness. Therefore, this work is based on the hypothesis that seed soaking in MGE enriched in PAs will mitigate drought stress by enhancing the growth, yield, and physio-biochemical systems of wheat plants. Thus, in this study, the effect of wheat seed priming in MGE (without or with enrichment by PAs) on growth and yield, physio-biochemical characteristics, including the up-regulation of AsA-GSH cycle, glyoxalase system, and PAs gene expression of wheat plants was studied under DIw stress conditions.

\section{Materials and Methods}

\subsection{Plant Material and Its Preparation for Sowing}

The experiment was carried out using certified Triticum aestivum (L.) seeds (cv. Giza168; winter wheat). Filtered calcium hypochlorite $(1 \%, v / v)$ was used to sterilize the seed surface for $1 \mathrm{~h}$. Then, the seeds were washed directly several times with sterile deionized water. The seeds were divided into three groups for different 6-h soaking treatments. The seeds of the first group were soaked in distilled water to serve as a control. The seeds of the second group were soaked in maize grain extract (MGE, 2\%) not enriched with polyamines (PAs). The seeds of the third group were soaked in MGE enriched with PAs (MGE A weight of $500 \mathrm{~g}$ of seeds was soaked in $1 \mathrm{~L}$ of each soaking solution, and the soaked seeds were re-dried under the shade with forced air according to the procedure of Sundstrom et al. [30]. The concentration 2\% was selected for both MGE and MGEPA based on our initial study carried out with 1,2, and 3\% of each of the extracts. Both levels 2 and 3\% conferred similar responses that were better than the response obtained from $1 \%$ in terms of wheat seedling growth (data not shown).

\subsection{Growing Conditions, Treatments, and Experimental Layout}

Black plastic pots ( $32 \mathrm{~cm}$ diameter and $30 \mathrm{~cm}$ depth) were used for this study. After sterilization, each pot was filled with $10 \mathrm{~kg}$ of pure sand, which was previously cleaned completely of ions with commercial acid and re-cleaned with distilled water. The sand was mixed with compost, vermicompost, and humic acid at a ratio of 90: 6.5: 3: 0.5 $(w / w)$, respectively. Ten uniform and healthy seeds were sown in each pot. The pots were arranged in a greenhouse as follows: the average temperatures were $19 \pm 3 / 10 \pm 2{ }^{\circ} \mathrm{C}$ for day (13 h)/night (11 h), and the average humidity was 62.0-65.1\%. The presence of sunlight inside the greenhouse remained homogeneous. Every three days, all pots were watered with full-strength nutritious solution ( $\mathrm{pH}$ 5.9) [31], containing $\mathrm{Ca}\left(\mathrm{NO}_{3}\right)_{2} \times 4 \mathrm{H}_{2} \mathrm{O}$, $\mathrm{KNO}_{3}, \mathrm{KH}_{2} \mathrm{PO}_{4}, \mathrm{MgSO}_{4} \times 7 \mathrm{H}_{2} \mathrm{O}, \mathrm{H}_{3} \mathrm{BO}_{3}, \mathrm{MnCl}_{2} \times 4 \mathrm{H}_{2} \mathrm{O}, \mathrm{ZnSO}_{4} \times 7 \mathrm{H}_{2} \mathrm{O}, \mathrm{CuSO}_{4} \times 5 \mathrm{H}_{2} \mathrm{O}$, $\mathrm{Na}_{2} \mathrm{MoO}_{4} \times 2 \mathrm{H}_{2} \mathrm{O}$, and $\mathrm{Fe}^{3+}{ }_{-} \mathrm{EDTA}^{+}$at the concentrations of $1.25 \mathrm{mM}, 1.25 \mathrm{mM}, 0.25 \mathrm{mM}$, $0.50 \mathrm{mM}, 11.6 \mu \mathrm{M}, 2.4 \mu \mathrm{M}, 0.24 \mu \mathrm{M}, 0.08 \mu \mathrm{M}, 0.13 \mu \mathrm{M}$, and $22.5 \mu \mathrm{M}$, respectively. Twenty days after sowing (DAS), water treatments were started with wheat seedlings of the three groups of soaking treatments. Each group of soaking treatments was assigned 45 pots and divided into three subgroups of 15 pots each. Each subgroup (e.g., control, MGE, and

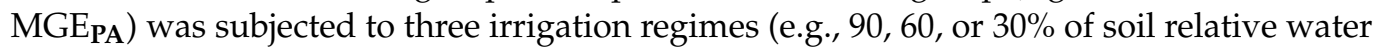
content) to include 9 treatments until harvest. The nutrient solution was applied along with the irrigation treatments, taking into account the equal concentration of nutrients in 
all pots for all treatments. Based on each water regime, soil moisture level was controlled by weighing each pot and any water loss was supplemented daily. A factorial completely randomized design was the one used to arrange all experimental pots. For the entire duration of the experiment (140 days), all pots were rotated daily to avoid systematic error due to fluctuations in environmental conditions.

\subsection{Calculation of Soil Relative Water Content (SRWC) and Preparation of Samples}

For the calculation of SRWC, the weighing method was used for the three water treatments (control (90\%), 60\%, or 30\% of SRWC). Daily, the amount of water transpired, and evaporated was compensated by the weight of the pots and watered to the corresponding target SRWC, which was expressed using the following equation:

$$
\text { SRWC }=\left(\mathrm{W}_{\text {soil }}-\mathrm{W}_{\text {pot }}-\mathrm{DW}_{\text {soil }}\right) /\left(\mathrm{W}_{\mathrm{FC}}-\mathrm{W}_{\text {pot }}-\mathrm{DW}_{\text {soil }}\right) \times 100
$$

where $W_{\text {soil }}$; current weight of soil, pot, and water, $W_{\text {pot }}$; empty pot weight, $D W_{\text {soil }}$; dry soil weight, and $W_{\mathrm{FC}}$; soil weight (soil + pot + water) at field capacity. Samples (the upper fully-expanded leaves) were taken 70 DAS (at the end of the tillering; vegetative growth stage with the first node) to determine the parameters of physiology, biochemistry, and antioxidative defense system components. Five plants (replicates) were functioned for each determination. Grain yield was obtained at the end of experiments (140 DAS) from 100 plants.

\subsection{Preparation of Maize Grain Extracts (MGE and $M G E_{P A}$ )}

The full method outlined in Alzahrani and Rady [25] and Alharby et al. [26] was used to obtain the extract from maize grains using local genotype of Egyptian maize. Wet cotton and clean cloth were used to cover and maintain the grains of maize until they were mushy. The mushy grains were transferred for milling using an appropriate amount of distilled water. Then, the mixture was filtered under vacuum. The filtrate was maintained in black bottles at $4{ }^{\circ} \mathrm{C}$ in a refrigerator. The remaining residue was transferred, quantitatively, for another extraction with methyl alcohol $(70 \%)$ for $72 \mathrm{~h}$, and the filtration process was repeated again. Using a rotary evaporator, the filtrate was completely evaporated to remove alcohol. The alcoholic extract was mixed well with the aqueous extract and, then, the mixture was concentrated to reach the target concentrations, which were used immediately. Otherwise, they were stored in a refrigerator at $-20{ }^{\circ} \mathrm{C}$ until use.

Some major ingredients in MGE were detected. AsA and GSH contents were determined in MGE according to Kampfenkel and Van Montagu [32] and Griffth [33], respectively. Activity of antioxidants was assayed in MGE by using the DPPH-radical scavenging activity [34]. Fresh extract was used for endogenous levels of phytohormones (e.g., auxins, gibberellins, cytokinins, including zeatin-type-cytokinins). The sample was frozen in liquid $\mathrm{N}$ for preparing to extract different phytohormones that were then analyzed using the GC/MS system according to Lavrich and Hays [35]. Polyamines (PAs) were extracted and determined as follows: Extraction of PAs was implemented at $4{ }^{\circ} \mathrm{C}$ by utilizing $500 \mathrm{mg}$ of top fresh fully (third and fourth)-expanded leaves with $4 \mathrm{~mL}$ fresh $5 \%(v / v) \mathrm{HClO}_{4}$. The supernatant obtained after centrifugation $(15,000 \times \mathrm{g}, 30 \mathrm{~min})$ was utilized to detect the free PAs (e.g., PUT, SPM, and SPD) using HPLC system [36-38]. Identification and quantification of PAs were conducted by comparing the retention times with peaks areas using the standards of PAs (observed on $254 \mathrm{~nm}$ using a 2487 dual UV-detector; Waters, Milford, MA, USA). Results of all assessments are shown in Table 1. As shown, the contents of PAs detected in MGE were very low $\left(0.5,0.6\right.$, and $0.9 \mu \mathrm{mol} \mathrm{g}^{-1} \mathrm{FW}$ for PUT, SPD, and SPM, respectively), and thus, MGE at $\%$ level was enriched with PUT, SPD, and SPM by adding them at rates of $0.5,0.6$, and $0.9 \mathrm{mM}$, respectively. These added concentrations of different PAs tested were based on their contents in MGE.

\subsection{Components of Growth and Yield}

Seventy DAS (tillering stage), five plants were selected and gently extracted from five randomly selected pots. The plants were gently cleaned from the sand particles by using 
tap water. After determining the FW of shoots, they were dried at $70{ }^{\circ} \mathrm{C}$ up to obtaining a constant DW. The grain yield components [Grain yield $\left(\mathrm{g} \mathrm{pot}^{-1}\right)$ and 1000-grain weight (g)] were assessed 140 DAS (harvest stage) by using 100 plants from each treatment.

\subsection{Leaf Pigments, Photosynthetic Efficiency, and Gas Exchange Parameters}

A weight of $0.2 \mathrm{~g}$ of fresh tissue of leaf was extracted using $80 \%(v / v)$ acetone with a clean mortar and pestle to determine chlorophylls and carotenoids [39]. Optical densities of supernatants were measured using spectrophotometer apparatus at wavelengths of 663, 645, and $480 \mathrm{~nm}$. Using top fresh leaves, the fluorometer apparatus (Hansatech Instr. Ltd., Kings Lynn, UK) was utilized to evaluate photosynthetic efficiency components; maximum quantum yield of PSII (Fv/Fm; [40]), photosynthetic performance index of PSII (PI ABS $_{\text {) was }}$ calculated [41], and hill reaction activity was measured [42]. Using the third upper leaf on five random plants, the infrared gas analyzer apparatus (LCA-4 model, England) was utilized to evaluate each net photosynthesis rate; $P n, \mathrm{CO}_{2}$ assimilation rate; $A$, conductance of leafy stomata; $g s$, and transpiration rate; $E$. Measurements were assessed 4 times $(2 \mathrm{~h}$ time interval from 7:00 to 17:00); 40, 50, 60, and 70 DAS.

\subsection{Oxidative Stress Biomarkers (Hydrogen Peroxide and Superoxide), and Peroxidation of Lipids}

Using the fresh upper leaves free of midribs from 5 randomly selected plants, the methods described in Velikova et al. [43], Kubis [44], and Madhava Rao and Sresty [45] were utilized to determine $\mathrm{H}_{2} \mathrm{O}_{2}, \mathrm{O}_{2}{ }^{\bullet-}$, and lipid peroxidation (evaluated as malondialdehyde; MDA) contents.

For $\mathrm{H}_{2} \mathrm{O}_{2}$, the content ( $\mu \mathrm{mol} \mathrm{g}{ }^{-1} \mathrm{FW}$ ) was evaluated colorimetrically by recording the absorbance readings of samples at $390 \mathrm{~nm}$ and the calculations were performed based on a suitable standard curve. For $\mathrm{O}_{2}{ }^{\bullet-}$, the content $\left(\mu \mathrm{mol} \mathrm{g}{ }^{-1} \mathrm{FW}\right)$ was evaluated using sample fragments $(1 \times 1 \mathrm{~mm}, 0.1 \mathrm{~g})$ that flooded using a $10 \mathrm{mM} \mathrm{K}$-phosphate buffer $(\mathrm{pH} 7.8)$, which was mixed with each of NBT $(0.05 \%)$ and $\mathrm{NaN}_{3}(10 \mathrm{mM})$ for $1 \mathrm{~h}$ at room temperature. The mixture was heated for $0.25 \mathrm{~h}$ on $85^{\circ} \mathrm{C}$. Then, the mixture was cooled rapidly. The absorbance readings were taken at $580 \mathrm{~nm}$. For MDA, the content was evaluated using the extinction coefficient $155 \mathrm{mM}^{-1} \mathrm{~cm}^{-1}$.

\subsection{Ion Leakage, Relative Water Content, and Membrane Stability Index}

Using fresh top leaves free of midribs from 5 randomly selected plants, the leaf ionic (electrolyte) leakage [46], leaf relative content of water [47], and stability index of cell membranes [46] were estimated.

\subsection{Assaying Enzymatic Activities and Ascorbate-Glutathione Cycle Activity}

Fresh top leaves free of midribs $(0.5 \mathrm{~g})$ from five plants were used to extract antioxidant enzymes for assaying the activities of ascorbate peroxidase (APX), superoxide dismutase (SOD), glutathione reductase (GR), and catalase (CAT). The methods of Dhindsa and Matowe [48], Aebi [49], Hasanuzzaman and Fujita [50], Nakano and Asada [51], Foster and Hess [52], Nakano and Asada [51], and Miyake and Asada [53] were employed to assay the activities of SOD (EC: 1.15.1.1), CAT (EC: 1.11.1.6), glutathione $S$-transferase (GST; EC: 2.5.1.18), APX (EC: 1.11.1.1), GR (EC: 1.6.4.2), DHAR (EC: 1.8.5.1), and MDHAR (EC: 1.6.5.4) in Unit $\mathrm{mg}^{-1}$ protein. Ascorbate (AsA) was determined in the tissue of upper fully expanded fresh leaves according to Huang et al. [54]. Determination of GSH pool was performed by applying the Yu et al. [55] method with a minor modification [56] with using a known concentration of both GSH and GSSG as standard curves.

\subsection{Glyoxalase System}

To determine the glyoxalase I (Gly I; EC: 4.4.1.5) and glyoxylase II (Gly II; EC: 3.1.2.6), the Hasanuzzaman and Fujita [50] method was applied using fresh top leaves free of midribs from five plants selected randomly. For Gly I, the mixture of assaying consisted of a buffer, pH 7.0 (100 mM K-phosphate), $\mathrm{MgSO}_{4}(15 \mathrm{mM}), \mathrm{GSH}(1.7 \mathrm{mM})$, and methylglyoxal 
(3.5 mM) in $900 \mu \mathrm{L}$ as a final volume. After addition of methylglyoxal, the changes occurred in the absorbance were observed at $240 \mathrm{~nm}$. For Gly II, a reaction mixture $(1.5 \mathrm{~mL})$ consisted of a buffer, pH 7.2 (100 mM Tris-HCl), $200 \mu \mathrm{M}$ of DTNB, and $1 \mathrm{mM}$ of S-D-lactoylglutathione was applied, and the extinction coefficients $3.37 \mathrm{mM}^{-1} \mathrm{~cm}^{-1}$ and $13.6 \mathrm{mM}^{-1} \mathrm{~cm}^{-1}$ were used to calculate the Gly I and Gly II, respectively.

\subsection{Proline, Glycinebetaine (GB), and Soluble Sugars (S. Sugars)}

Using fresh top leaves free of midribs from five plants selected randomly and toluene, the extraction of proline was practiced. At $520 \mathrm{~nm}$, the absorbance was recorded [57]. Leaf content ( $\mu \mathrm{g}$ proline $\mathrm{g}^{-1} \mathrm{FW}$ ) of proline was calculated using a suitable standard curve. The Grieve and Grattan [58] method was applied to estimate GB. The formed periodide crystals were monitored colorimetrically at $365 \mathrm{~nm}$ after reacting the mixture with cold KI-I 2 (a reagent) under acidic condition. The method of Irigoyen et al. [59] was utilized for extracting (using ethanol, 96\%) and determining the content of soluble sugars (S. sugars) in $\mathrm{mg} \mathrm{g}^{-1}$ dry leafy mass (DW). The ethanolic extract $(100 \mu \mathrm{L})$ was reacted with $150 \mathrm{mg}$ of anthrone reagent (freshly prepared in $100 \mathrm{~mL} \mathrm{H}_{2} \mathrm{SO}_{4}, 72 \%$ ), and the mixture was then boiled for $10 \mathrm{~min}$ in a water bath. Colorimetrically, the absorbance readings were recorded at $625 \mathrm{~nm}$ after cooling.

2.12. Polyamines (PAs) Content Determinations and Relative Expression of Biosynthetic Genes of PAs Relative Expression (Using qRT-PCR)

Chen et al. [36], Guo et al. [37], and Flores and Galston [38] procedures were used with the HPLC system and $0.5 \mathrm{~g}$ fresh leaves for extraction of PAs at $4{ }^{\circ} \mathrm{C}$. After a centrifugation process $(15,000 \times g, 30 \mathrm{~min})$, the supernatant was subjected to detect PuT, SpM, and SpD. Retention times were compared with the peak areas (noticed on $254 \mathrm{~nm}$ using a 2487 dual UV-detector; Waters, Milford, MA, USA) obtained to identify and quantify the three PAs.

According to the manufacturer's protocol, RNA isolation (using TRIsure; Bioline, London, UK), cDNA synthesis, and quantitative real-time analysis (qRT-PCR) were implemented with $0.1 \mathrm{~g}$ of fresh leaves. DNase I (Thermo Scientific, London, UK) was utilized to digest RNA samples, and then RNA was spectrophotometerically quantified. Using Agarose gel electrophoresis, the determination of overall purity and integrity of RNA was conducted. For the reverse-transcription of RNA to cDNA, $1 \mu \mathrm{g}$ of total RNA was used with a kit of Sensifast first cDNA synthesis (Bioline, London, UK). Based on the NCBI, the designation of primers and the Genome Database of wheat were as follows: ADC (F: 5'-CAACGACTTTGTTAGCTTTGG-3' , R: 5'-CAGGCTTGGCTTTGGTAA-3'), ODC (F: 5'-GGCCACTTCTTCTAGGTTCA-3', R: 5'-ACTCGGCGTCTTATATAGCG-3'), SAMDC (F: 5'-CGAGCTTGTGTTGCGTCAG-3', R: 5' -ATACATTCGCTCACACTGGCA-3'), SPDS (F: $5^{\prime}$ CTGAGAGTATGTGGTTGCAT-3', R: 5'-CATAGTGGACAGAACCCTTG-3'), SPMS (F: 5' AGTAGAGAAGATTTTGTACCAGG-3', R: 5'-GGACATTCCCATAGGTTGAAG-3'), DHS (F: 5'-TCACTCGGAGACATGCTGTT-3', R: 5'-CAGCCTTATATCTTGTACAATGTCG-3'), and GAPDH (F: 5' -TTGCTCTGAACGACCATTTC-3' ${ }^{\prime}$, R: 5'-GACACCATCCACATTTATTC TTC-3').

Using diluted cDNA samples, the qPCR was implemented in a $20 \mathrm{~mL}$ reaction mixture (10 mL of SensiFast SYBR Lo-Rox 2X mix (Bioline, London, UK) with $1.2 \mathrm{~mL}$ (300 nM) of each primer). The PCR was implemented by using a STRATAGENE MxPro-3000P (Agilent Technologies). Calculation of relative expression was done by using the method of 2-DDCt, where the level of mRNA relative expression was normalized versus the internal standard gene (GAPDH) and was then compared with control.

\subsection{Zeatin-Type Cytokinin}

Fresh leaf blade was frozen in liquid N, grounded, and extracted for trans-zeatin-type cytokinin; $t-Z$ and cis-zeatin-type cytokinin; $c-Z$. The analysis was performed according to the Novák et al. [60] and Forcat et al. [61] procedures. 


\subsection{Experimental Design and Statistical Analysis}

The experiment was repeated three times and carried out as a factorial completely randomized design with three soaking treatments (distilled water, $\%$ MGE, or $2 \% \mathrm{MGE}_{\mathbf{P A}}$ ) and three irrigation levels (90\% as a control, $60 \%$, or $30 \%$ of SRWC) in 15 replications (pots). Data are means ( \pm standard error; SE). Testing for homogeneity of error variances was conducted according to the procedure outlined by Gomez and Gomez [62]. Combined analysis of data of the three experiments was conducted, and statistical analysis of all data was performed by using Statistica (version 9, Tulsa, OK, USA), subjected to two-way ANOVA, and differences among treatment means were affirmed statistically by using the Fisher LSD test at $p \leq 0.05$.

\section{Results}

\subsection{Growth, Yield, Leaf Photosynthetic Pigments, and Photosynthetic Efficiency}

The treatment Ir60\% (moderate drought) or Ir30\% (severe drought) markedly decreased wheat plant growth (shoot fresh weight and shoot dry weight), yield (grain yield and 1000-grain weight), leaf pigments (total chlorophylls and total carotenoids), and photosynthetic efficiency (PSII efficiency; Fv / Fm, PSII performance index, and hill reaction activity; HRA) compared to Ir90\% (optimal irrigation) (Figures 1 and 2). Severe effect was obtained with Ir $30 \%$, which did not give yield because the plants did not survive. However, under Ir $90 \%$ or Ir60\%, seed soaking in 2\% MGE or MGE PA significantly increased plant growth, yield, leaf pigments, and photosynthetic efficiency compared to the corresponding controls. MGE $\mathrm{PA}_{\mathrm{PA}}$ was more efficient than MGE, and the tested parameters responded better to MGE or MGE $\mathrm{PA}_{\mathrm{PA}}$ under $\operatorname{Ir} 60 \%$ than $\operatorname{Ir} 90 \%$. On the other hand, under Ir $30 \%$, either MGE or $\mathrm{MGE}_{\mathrm{PA}}$ failed to increase the parameters mentioned above compared to the corresponding control. The interaction (drought $\times$ MGE) was significant ( $p \leq 0.05$ or 0.01 ) for all tested attributes (Figures 1 and 2).

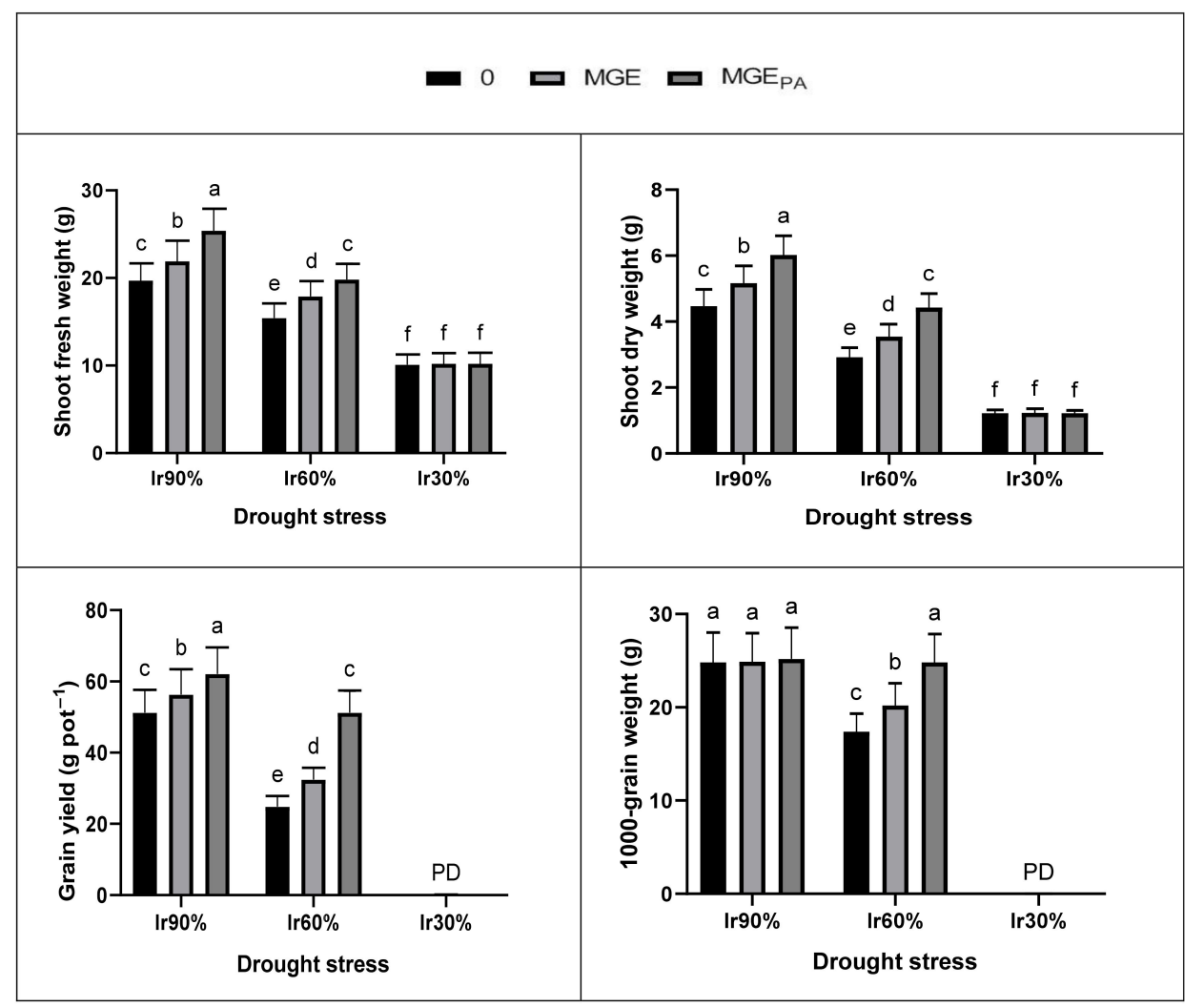

Figure 1. Positive seed priming impacts using maize grain extract without (MGE, $\%$ ) or with polyamines enrichment $\left(\mathrm{MGE}_{\mathrm{PA}}, 2 \%\right)$ on growth and yield components of drought-stressed wheat plants. 


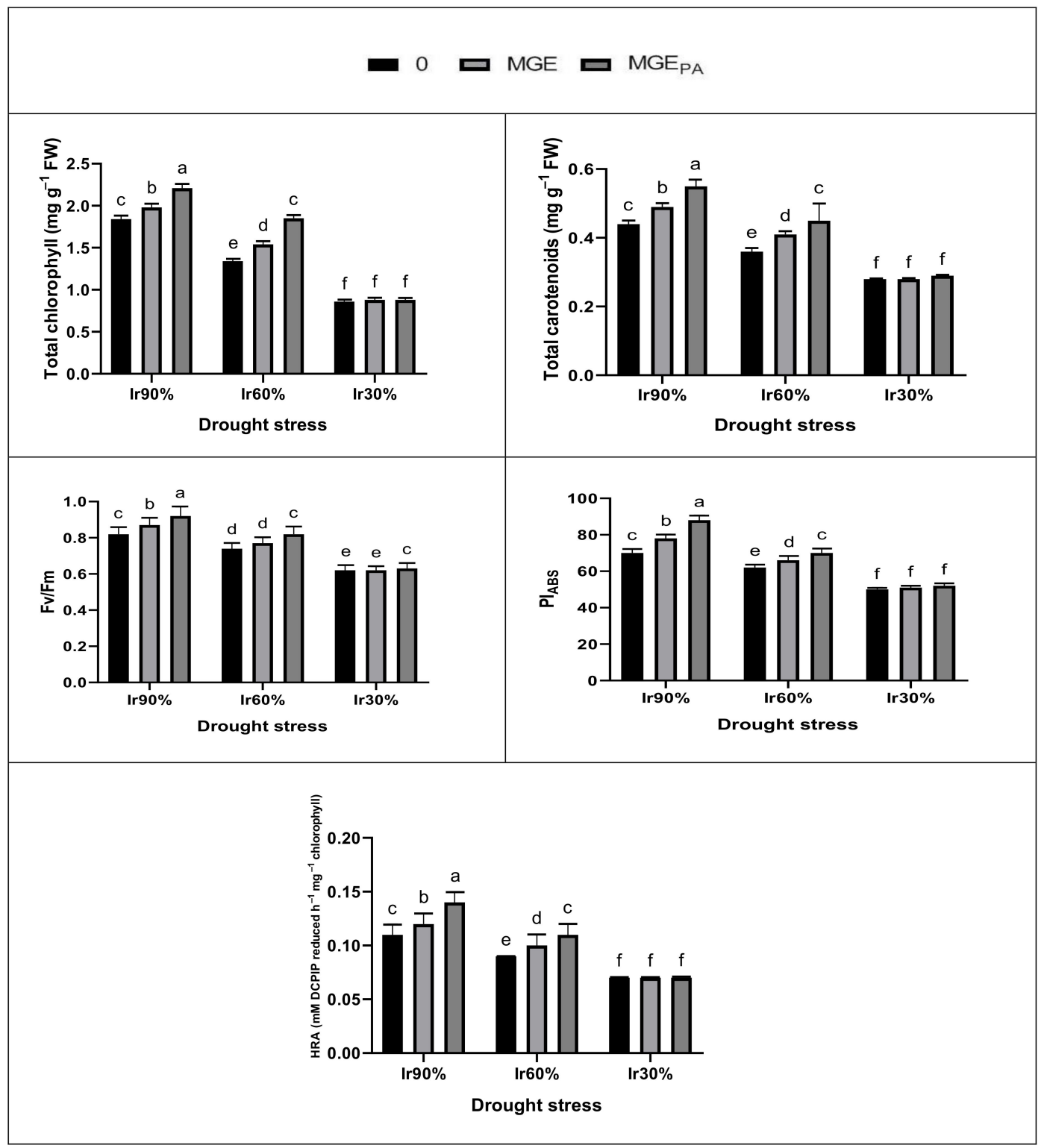

Figure 2. Positive seed priming impacts using maize grain extract without (MGE, $\%$ ) or with polyamines enrichment $\left(\mathrm{MGE}_{\mathrm{PA}}, 2 \%\right)$ on leaf photosynthetic pigments, and chlorophyll fluorescence of drought-stressed wheat plants.

\subsection{Gas Exchange Parameters and Plant Leaf Tissue Health}

Application of moderate or severe drought noticeably decreased gas exchange parameters (Pn, A, gs, and E), MSI, and RWC of wheat plants compared to optimal irrigation (Table 2). Ir $30 \%$ treatment caused higher reductions than Ir60\%. However, under Ir $90 \%$ or Ir60\%, seed soaking with MGE or MGE while MSI and RWC were only increased under Ir60\% compared to the corresponding control. MGE PA was more efficient than MGE, and the tested parameters responded better to MGE or MGE increase the tested parameters under Ir30\% compared to the corresponding control. The interaction (drought $\times$ MGE) was significant ( $p \leq 0.05$ or 0.01 ) for all tested attributes (Table 2). 
Table 2. Positive seed priming impacts using maize grain extract without (MGE, $2 \%$ ) or with polyamines enrichment $\left(\mathrm{MGE}_{\mathrm{PA}}, 2 \%\right)$ on gas exchange parameters of drought-stressed wheat plants.

\begin{tabular}{|c|c|c|c|c|c|}
\hline \multicolumn{2}{|c|}{ Treatments } & \multicolumn{4}{|c|}{ Parameters } \\
\hline Drought & MGE \% & $\begin{array}{l}P n(\mu \mathrm{mol} \\
\left.\mathrm{m}^{-1} \mathrm{~S}^{-1}\right)\end{array}$ & $\begin{array}{c}A(\mathrm{mmol} \mathrm{CO} \\
\left.\mathrm{m}^{-1} \mathrm{~S}^{-1}\right)\end{array}$ & $\begin{array}{c}g s\left(\mathrm{mmol} \mathrm{CO}_{2}\right. \\
\left.\mathrm{m}^{-1} \mathrm{~S}^{-1}\right)\end{array}$ & $\underset{\left.\mathrm{m}^{-1} \mathrm{~S}^{-1}\right)}{E\left(\mathrm{mmol} \mathrm{H}_{2} \mathrm{O}\right.}$ \\
\hline \multirow{4}{*}{$\operatorname{Ir} 90 \%$} & 0 & $11.6 \pm 0.3 c$ & $14.5 \pm 0.4 b$ & $327 \pm 14 \mathrm{c}$ & $1.8 \pm 0.08 \mathrm{c}$ \\
\hline & MGE & $12.1 \pm 0.3 \mathrm{~b}$ & $15.5 \pm 0.4 \mathrm{a}$ & $352 \pm 15 b$ & $2.0 \pm 0.09 \mathrm{~b}$ \\
\hline & MGE $_{P A}$ & $12.8 \pm 0.4 \mathrm{a}$ & $15.6 \pm 0.5 \mathrm{a}$ & $390 \pm 17 a$ & $2.3 \pm 0.11 \mathrm{a}$ \\
\hline & 0 & $9.5 \pm 0.2 \mathrm{e}$ & $11.2 \pm 0.3 \mathrm{~d}$ & $208 \pm 10 \mathrm{e}$ & $1.1 \pm 0.05 \mathrm{e}$ \\
\hline \multirow[t]{3}{*}{$\operatorname{Ir} 60 \%$} & MGE & $10.4 \pm 0.2 \mathrm{~d}$ & $12.6 \pm 0.4 \mathrm{c}$ & $261 \pm 12 \mathrm{~d}$ & $1.4 \pm 0.07 \mathrm{~d}$ \\
\hline & MGE $_{P A}$ & $11.6 \pm 0.3 c$ & $14.4 \pm 0.5 b$ & $328 \pm 14 \mathrm{c}$ & $1.8 \pm 0.09 c$ \\
\hline & 0 & $4.8 \pm 0.1 \mathrm{f}$ & $6.7 \pm 0.2 \mathrm{e}$ & $112 \pm 6 \mathrm{f}$ & $0.5 \pm 0.02 \mathrm{f}$ \\
\hline \multirow[t]{2}{*}{$\operatorname{Ir} 30 \%$} & MGE & $4.8 \pm 0.1 \mathrm{f}$ & $6.8 \pm 0.2 \mathrm{e}$ & $114 \pm 6 \mathrm{f}$ & $0.5 \pm 0.02 \mathrm{f}$ \\
\hline & MGE $_{\mathrm{PA}}$ & $4.9 \pm 0.1 \mathrm{f}$ & $6.8 \pm 0.2 \mathrm{e}$ & $116 \pm 6 \mathrm{f}$ & $0.5 \pm 0.02 \mathrm{f}$ \\
\hline \multicolumn{6}{|c|}{ Two way ANOVA results } \\
\hline \multicolumn{2}{|c|}{ Drought (D) } & $(* *)$ & $\left({ }^{*}\right)$ & $(* *)$ & $(*)$ \\
\hline \multicolumn{2}{|c|}{ MGE } & $(*)$ & $(*)$ & $(*)$ & $(*)$ \\
\hline \multicolumn{2}{|c|}{$\mathrm{D} \times \mathrm{MGE}$} & $(*)$ & $(*)$ & $\left({ }^{*}\right)$ & $(*)$ \\
\hline
\end{tabular}

Values presented within columns are means $(n=5) \pm$ standard error. The experiment was repeated three times. Means followed by different letter are significantly different $(p \leq 0.05)$. $\left.{ }^{* *}\right)$ or $\left(^{*}\right)$ significant at 1 or $5 \%$ probability levels, respectively. $\mathrm{Pn}$ means net photosynthesis rate, $A$ means $\mathrm{CO}_{2}$ assimilation rate, $g s$ means stomatal conductance, and $E$ means transpiration rate.

\subsection{Oxidative Stress Levels, Lipid Peroxidation, and Electrolyte Leakage (EL)}

Compared to $\operatorname{Ir} 90 \%$, Ir $30 \%$ treatment noticeably exceeded Ir $60 \%$ in increasing oxidative stress biomarkers $\left(\mathrm{O}_{2}{ }^{\bullet-}\right.$ and $\left.\mathrm{H}_{2} \mathrm{O}_{2}\right)$, lipid peroxidation (assessed as malondialdehyde; MDA accumulation), and EL of wheat plants (Table 3). The tested parameters did not

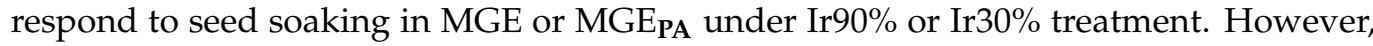
under Ir60\%, MGE EL levels compared to the corresponding control. The interaction (drought $\times \mathrm{MGE}$ ) was significant $(p \leq 0.05$ or 0.01$)$ for all tested parameters (Table 3 ).

Table 3. Positive seed priming impacts using maize grain extract without (MGE, $2 \%$ ) or with polyamines enrichment $\left(\mathrm{MGE}_{\mathrm{PA}}, 2 \%\right.$ ) on oxidative stress levels (superoxide; $\mathrm{O}_{2}{ }^{--}$and hydrogen peroxide; $\mathrm{H}_{2} \mathrm{O}_{2}$ ), malondialdehyde (MDA) accumulation, electrolyte leakage (EL), membrane stability index (MSI), and relative water content (RWC) of droughtstressed wheat plants.

\begin{tabular}{|c|c|c|c|c|c|c|c|}
\hline \multicolumn{2}{|c|}{ Treatments } & \multicolumn{6}{|c|}{ Parameters } \\
\hline Drought & MGE \% & $\begin{array}{c}\mathrm{H}_{2} \mathrm{O}_{2}(\mu \mathrm{mol} \\
\left.\mathrm{g}^{-1} \mathrm{FW}\right)\end{array}$ & $\begin{array}{c}\mathrm{O}_{2}^{\bullet-}(\mu \mathrm{mol} \\
\left.\mathrm{g}^{-1} \mathrm{FW}\right)\end{array}$ & $\begin{array}{c}\text { MDA ( } \mu \text { mol } \\
\mathrm{g}^{-1} \text { FW) }\end{array}$ & EL (\%) & MSI (\%) & RWC (\%) \\
\hline \multirow{4}{*}{$\operatorname{Ir} 90 \%$} & 0 & $3.52 \pm 0.05 \mathrm{~d}$ & $1.21 \pm 0.01 \mathrm{~d}$ & $10.24 \pm 0.11 \mathrm{~d}$ & $6.12 \pm 0.25 \mathrm{~d}$ & $64.4 \pm 1.4 \mathrm{a}$ & $73.6 \pm 1.9 \mathrm{a}$ \\
\hline & MGE & $3.50 \pm 0.05 \mathrm{~d}$ & $1.20 \pm 0.01 \mathrm{~d}$ & $10.20 \pm 0.11 \mathrm{~d}$ & $6.11 \pm 0.24 \mathrm{~d}$ & $64.9 \pm 1.4 \mathrm{a}$ & $74.1 \pm 2.0 \mathrm{a}$ \\
\hline & $\mathrm{MGE}_{\mathrm{PA}}$ & $3.50 \pm 0.05 \mathrm{~d}$ & $1.20 \pm 0.01 \mathrm{~d}$ & $10.18 \pm 0.10 \mathrm{~d}$ & $6.10 \pm 0.24 \mathrm{~d}$ & $65.4 \pm 1.5 \mathrm{a}$ & $75.0 \pm 1.9 \mathrm{a}$ \\
\hline & 0 & $5.43 \pm 0.08 b$ & $2.54 \pm 0.03 b$ & $18.14 \pm 0.21 b$ & $14.52 \pm 0.51 b$ & $46.5 \pm 1.3 c$ & $52.4 \pm 1.4 \mathrm{c}$ \\
\hline \multirow[t]{3}{*}{ Ir60\% } & MGE & $4.66 \pm 0.07 c$ & $1.79 \pm 0.02 c$ & $15.05 \pm 0.16 c$ & $11.02 \pm 0.42 c$ & $58.8 \pm 1.4 b$ & $64.0 \pm 1.6 b$ \\
\hline & MGE $_{P A}$ & $3.48 \pm 0.05 \mathrm{~d}$ & $1.20 \pm 0.01 \mathrm{~d}$ & $10.19 \pm 0.10 \mathrm{~d}$ & $6.14 \pm 0.26 \mathrm{~d}$ & $64.2 \pm 1.4 \mathrm{a}$ & $73.5 \pm 1.9 \mathrm{a}$ \\
\hline & 0 & $9.54 \pm 0.14 \mathrm{a}$ & $4.22 \pm 0.05 \mathrm{a}$ & $32.41 \pm 0.35 a$ & $36.26 \pm 1.24 \mathrm{a}$ & $30.1 \pm 0.9 \mathrm{~d}$ & $34.2 \pm 1.2 \mathrm{~d}$ \\
\hline \multirow[t]{2}{*}{$\operatorname{Ir} 30 \%$} & MGE & $9.51 \pm 0.16 \mathrm{a}$ & $4.20 \pm 0.05 \mathrm{a}$ & $32.36 \pm 0.35 a$ & $36.21 \pm 1.22 \mathrm{a}$ & $30.4 \pm 0.8 \mathrm{~d}$ & $34.5 \pm 1.4 \mathrm{~d}$ \\
\hline & MGE $_{\mathrm{PA}}$ & $9.49 \pm 0.13 \mathrm{a}$ & $4.20 \pm 0.05 \mathrm{a}$ & $32.34 \pm 0.35 \mathrm{a}$ & $36.20 \pm 1.18 \mathrm{a}$ & $31.3 \pm 0.9 \mathrm{~d}$ & $35.1 \pm 1.2 \mathrm{~d}$ \\
\hline \multicolumn{8}{|c|}{ Two way ANOVA results } \\
\hline \multicolumn{2}{|c|}{ Drought (D) } & $(*)$ & $(* *)$ & $(*)$ & $(* *)$ & $(*)$ & $(*)$ \\
\hline \multicolumn{2}{|c|}{ MGE } & $\left({ }^{*}\right)$ & $(*)$ & $\left({ }^{*}\right)$ & $(* *)$ & $(*)$ & $\left({ }^{*}\right)$ \\
\hline \multicolumn{2}{|c|}{$\mathrm{D} \times \mathrm{MGE}$} & $(*)$ & $(*)$ & $(*)$ & $(* *)$ & $(*)$ & $(*)$ \\
\hline
\end{tabular}

Values presented within columns are means $(n=5) \pm$ standard error. The experiment was repeated three times. Means followed by different letter are significantly different $(p \leq 0.05) .\left({ }^{*}\right)$ or $\left.{ }^{*}\right)$ significant at 1 or $5 \%$ probability levels, respectively. 


\subsection{Activities of Antioxidant Enzymes and Ascorbate-Glutathione (AsA-GSH) Cycle}

Ir $30 \%$ treatment markedly decreased the activities of antioxidant enzymes and AsAGSH cycle (SOD, CAT, GST, AsA, APX, GR, MDHAR, DHAR, GSH, and GSSG), while Ir60\% treatment considerably increased the activities of these antioxidant enzymes and AsA-GSH cycle, except for the activities of AsA, MDHAR, and DHAR activities, which were further decreased in wheat plants compared to Ir90\% (Tables 4 and 5). These tested activities of antioxidant enzymes and AsA-GSH cycle did not respond to seed soaking in MGE or MGE $_{\mathbf{P A}}$ under Ir $90 \%$ or Ir $30 \%$ treatment. However, under Ir60\%, $\mathrm{MGE}_{\mathbf{P A}}$ significantly exceeded MGE, both of which significantly elevated the activities of antioxidant enzymes and AsA-GSH cycle compared to the corresponding control. The interaction (drought $x$ MGE) was significant ( $P \leq 0.05$ or 0.01 ) for all tested parameters (Tables 4 and 5 ).

Table 4. Positive seed priming impacts using maize grain extract without (MGE, $2 \%$ ) or with polyamines enrichment $\left(\mathrm{MGE}_{\mathrm{PA}}, 2 \%\right)$ on the activities of antioxidant enzymes of drought-stressed wheat plants.

\begin{tabular}{|c|c|c|c|c|}
\hline \multicolumn{2}{|c|}{ Treatments } & \multicolumn{3}{|c|}{ Parameters } \\
\hline Drought & MGE \% & $\begin{array}{l}\text { SOD (EU mg }{ }^{-1} \\
\text { Protein) }\end{array}$ & $\begin{array}{c}\text { CAT (EU mg } \text { m }^{-1} \\
\text { Protein) }\end{array}$ & $\begin{array}{c}\text { GST (EU mg } \text { m }^{-1} \\
\text { Protein) }\end{array}$ \\
\hline \multirow{4}{*}{$\operatorname{Ir} 90 \%$} & 0 & $45.4 \pm 0.5 \mathrm{~d}$ & $30.6 \pm 0.4 \mathrm{~d}$ & $17.8 \pm 0.2 \mathrm{~d}$ \\
\hline & MGE & $45.4 \pm 0.5 \mathrm{~d}$ & $30.6 \pm 0.4 \mathrm{~d}$ & $17.8 \pm 0.2 \mathrm{~d}$ \\
\hline & $\mathrm{MGE}_{\mathrm{PA}}$ & $45.5 \pm 0.5 \mathrm{~d}$ & $30.6 \pm 0.4 \mathrm{~d}$ & $17.9 \pm 0.2 \mathrm{~d}$ \\
\hline & 0 & $62.4 \pm 0.7 c$ & $41.2 \pm 0.4 c$ & $20.8 \pm 0.2 c$ \\
\hline \multirow[t]{2}{*}{$\operatorname{Ir} 60 \%$} & MGE & $67.2 \pm 0.7 b$ & $47.9 \pm 0.5 \mathrm{~b}$ & $25.2 \pm 0.3 b$ \\
\hline & MGEPA & $75.8 \pm 0.8 \mathrm{a}$ & $54.9 \pm 0.6 \mathrm{a}$ & $29.2 \pm 0.3 \mathrm{a}$ \\
\hline \multirow{3}{*}{$\operatorname{Ir} 30 \%$} & 0 & $29.4 \pm 0.3 \mathrm{e}$ & $18.7 \pm 0.2 \mathrm{e}$ & $11.8 \pm 0.1 \mathrm{e}$ \\
\hline & MGE & $29.5 \pm 0.3 \mathrm{e}$ & $18.7 \pm 0.2 \mathrm{e}$ & $11.8 \pm 0.1 \mathrm{e}$ \\
\hline & $\mathrm{MGE}_{\mathrm{PA}}$ & $29.4 \pm 0.3 \mathrm{e}$ & $18.8 \pm 0.2 \mathrm{e}$ & $11.9 \pm 0.2 \mathrm{e}$ \\
\hline \multicolumn{5}{|c|}{ Two way ANOVA results } \\
\hline \multicolumn{2}{|c|}{ Drought (D) } & $(*)$ & $(*)$ & $(*)$ \\
\hline \multicolumn{2}{|c|}{ MGE } & $\left({ }^{*}\right)$ & $\left({ }^{*}\right)$ & $(*)$ \\
\hline \multicolumn{2}{|c|}{$\mathrm{D} \times \mathrm{MGE}$} & $\left({ }^{*}\right)$ & $\left({ }^{*}\right)$ & $(*)$ \\
\hline
\end{tabular}

Values presented within columns are means $(n=5) \pm$ SE. The experiment was repeated three times. Means followed by different letter are significantly different $(p \leq 0.05) .\left({ }^{*}\right)$ significant at $5 \%$ probability levels, respectively. SOD means superoxide dismutase, CAT means catalase, and GST means glutathione-S-transferase.

Table 5. Positive seed priming impacts using maize grain extract without (MGE, $2 \%$ ) or with polyamines enrichment $\left(\mathrm{MGE}_{\mathrm{PA}}, 2 \%\right)$ on the activity of ascorbate-glutathione cycle of drought-stressed wheat plants.

\begin{tabular}{|c|c|c|c|c|c|c|c|c|}
\hline \multicolumn{2}{|c|}{ Treatments } & \multicolumn{7}{|c|}{ Parameters } \\
\hline Drought & MGE \% & $\begin{array}{c}\text { AsA ( } \mu \text { mol } \\
\left.\mathrm{g}^{-1} \mathrm{FW}\right)\end{array}$ & $\begin{array}{c}\text { APX (EU } \\
\text { mg }^{-1} \text { Protein) }\end{array}$ & $\begin{array}{l}\text { GR (EU } \\
\text { mg }^{-1} \\
\text { Protein) }\end{array}$ & $\begin{array}{l}\text { MDHAR } \\
\text { (EU mg }^{-1} \\
\text { Protein) }\end{array}$ & $\begin{array}{c}\text { DHAR (EU } \\
\text { mg }^{-1} \\
\text { Protein) }\end{array}$ & $\begin{array}{c}\text { GSH ( } \mu \mathrm{mol} \\
\left.\mathrm{g}^{-1} \mathrm{FW}\right)\end{array}$ & $\begin{array}{c}\text { GSSG } \\
\left(\mu \mathrm{mol} \mathrm{g}{ }^{-1}\right. \\
\text { FW) }\end{array}$ \\
\hline \multirow{4}{*}{$\operatorname{Ir} 90 \%$} & 0 & $4.3 \pm 0.06 b$ & $9.2 \pm 0.11 \mathrm{~d}$ & $6.7 \pm 0.10 \mathrm{~d}$ & $59.4 \pm 0.8 \mathrm{a}$ & $132 \pm 2 \mathrm{a}$ & $89 \pm 2 d$ & $22.2 \pm 0.4 \mathrm{~d}$ \\
\hline & MGE & $4.3 \pm 0.06 b$ & $9.2 \pm 0.11 \mathrm{~d}$ & $6.7 \pm 0.10 \mathrm{~d}$ & $59.6 \pm 0.8 \mathrm{a}$ & $132 \pm 2 \mathrm{a}$ & $89 \pm 2 d$ & $22.2 \pm 0.4 \mathrm{~d}$ \\
\hline & MGE $_{P A}$ & $4.4 \pm 0.06 \mathrm{~b}$ & $9.3 \pm 0.12 \mathrm{~d}$ & $6.8 \pm 0.11 \mathrm{~d}$ & $59.9 \pm 0.9 \mathrm{a}$ & $133 \pm 2 a$ & $90 \pm 2 d$ & $22.4 \pm 0.4 \mathrm{~d}$ \\
\hline & 0 & $3.2 \pm 0.04 c$ & $15.4 \pm 0.21 c$ & $8.4 \pm 0.14 c$ & $31.4 \pm 0.5 c$ & $72 \pm 1 c$ & $106 \pm 2 c$ & $41.2 \pm 0.7 c$ \\
\hline \multirow[t]{3}{*}{$\operatorname{Ir} 60 \%$} & MGE & $4.3 \pm 0.05 b$ & $17.2 \pm 0.24 b$ & $9.1 \pm 0.16 b$ & $42.7 \pm 0.6 b$ & $98 \pm 2 b$ & $124 \pm 3 b$ & $51.9 \pm 0.9 b$ \\
\hline & MGEPA $_{P A}$ & $4.8 \pm 0.07 \mathrm{a}$ & $19.7 \pm 0.30 \mathrm{a}$ & $9.9 \pm 0.18 \mathrm{a}$ & $58.9 \pm 0.8 \mathrm{a}$ & $129 \pm 2 \mathrm{a}$ & $149 \pm 3 \mathrm{a}$ & $63.5 \pm 1.1 \mathrm{a}$ \\
\hline & 0 & $2.4 \pm 0.03 \mathrm{~d}$ & $4.9 \pm 0.07 \mathrm{e}$ & $3.5 \pm 0.04 \mathrm{e}$ & $22.2 \pm 0.3 \mathrm{~d}$ & $50 \pm 1 \mathrm{~d}$ & $64 \pm 1 \mathrm{e}$ & $14.3 \pm 0.2 \mathrm{e}$ \\
\hline \multirow[t]{2}{*}{$\operatorname{Ir} 30 \%$} & MGE & $2.4 \pm 0.03 \mathrm{~d}$ & $4.9 \pm 0.07 \mathrm{e}$ & $3.6 \pm 0.05 \mathrm{e}$ & $22.2 \pm 0.3 \mathrm{~d}$ & $51 \pm 1 d$ & $64 \pm 1 \mathrm{e}$ & $14.4 \pm 0.2 \mathrm{e}$ \\
\hline & $\mathrm{MGE}_{\mathrm{PA}}$ & $2.5 \pm 0.03 \mathrm{~d}$ & $5.0 \pm 0.08 \mathrm{e}$ & $3.6 \pm 0.05 \mathrm{e}$ & $22.3 \pm 0.3 \mathrm{~d}$ & $52 \pm 1 \mathrm{~d}$ & $66 \pm 1 \mathrm{e}$ & $14.6 \pm 0.2 \mathrm{e}$ \\
\hline \multicolumn{9}{|c|}{ Two way ANOVA results } \\
\hline \multicolumn{2}{|c|}{ Drought (D) } & $\left({ }^{*}\right)$ & $(*)$ & $(*)$ & $(*)$ & $(*)$ & $\left({ }^{*}\right)$ & $\left({ }^{*}\right)$ \\
\hline \multicolumn{2}{|c|}{ MGE } & $(*)$ & $(*)$ & $(*)$ & $(*)$ & $(*)$ & $(*)$ & $(*)$ \\
\hline \multicolumn{2}{|c|}{$\mathrm{D} \times \mathrm{MGE}$} & $(*)$ & $(*)$ & $(*)$ & $(*)$ & $(*)$ & $(*)$ & $(*)$ \\
\hline
\end{tabular}

Values presented within columns are means $(n=5) \pm$ SE. The experiment was repeated three times. Means followed by different letter are significantly different $(p \leq 0.05)$. (*) significant at $5 \%$ probability levels, respectively. AsA means ascorbate, APX means ascorbate peroxidase, GR means glutathione reductase, MDHAR means monodehydro-ascorbate reductase, DHAR means dehydro-ascorbate reductase, GSH means glutathione, and GSSG means oxidized glutathione. 


\subsection{Glyoxalase System (Gly I and Gly II), Osmoprotectants, and Zeatins}

Compared with Ir90\%, Ir60\% noticeably increased Gly I activity, and osmoprotectants (proline, GB, and S. sugars), $t-Z$ and $c-Z$ contents, except for Gly II activity, which was decreased, while Ir $30 \%$ markedly decreased all parameters of wheat plants (Table 6). The parameters mentioned above did not respond to seed soaking in MGE or MGE under Ir $90 \%$ or Ir $30 \%$ treatment, except for trans-zeatin and cis-zeatin contents, which were markedly increased by pretreatment with MGE or MGE efficacy of MGE $_{\mathbf{P A}}$ than MGE. However, under Ir60\%, MGE $_{\mathbf{P A}}$ significantly exceeded MGE in increasing the activities and contents of all parameters mentioned above compared to the corresponding controls. The interaction (drought $\times$ MGE) was significant ( $p \leq 0.05$ or 0.01) for all tested parameters (Table 6).

Table 6. Positive seed priming impacts using maize grain extract without (MGE, $2 \%$ ) or with polyamines enrichment (MGE $\mathrm{PA}_{2}$ \%) on glyoxalase system (Gly I and Gly II), osmoprotectants (e.g., proline, glycine betaine; GB, and soluble sugars; S. sugars), trans- and cis-zeatin accumulation in drought-stressed wheat plants.

\begin{tabular}{|c|c|c|c|c|c|c|c|c|}
\hline \multicolumn{2}{|c|}{ Treatments } & \multicolumn{7}{|c|}{ Parameters } \\
\hline Drought & MGE \% & $\begin{array}{c}\text { Gly I (mmol } \\
\text { min }^{-1} \text { mg }^{-1} \\
\text { Protein) }\end{array}$ & $\begin{array}{c}\text { GlyII (mmol } \\
\text { min }^{-1} \mathrm{mg}^{-1} \\
\text { Protein) }\end{array}$ & $\begin{array}{l}\text { Proline ( } \mu \mathrm{g} \\
\left.\mathrm{g}^{-1} \mathrm{FW}\right)\end{array}$ & $\begin{array}{c}\text { GB }\left(\mu g g^{-1}\right. \\
\text { FW) }\end{array}$ & $\begin{array}{c}\text { Soluble } \\
\text { Sugars (mg } \\
\left.\mathrm{g}^{-1} \mathrm{FW}\right)\end{array}$ & $\begin{array}{l}\text { Trans-Zeatin } \\
\left.\text { (ng g }{ }^{-1} \mathrm{DW}\right)\end{array}$ & $\begin{array}{c}\text { Cis-Zeatin } \\
\left(\mathrm{ng} \mathrm{g}^{-1} \mathrm{DW}\right)\end{array}$ \\
\hline \multirow{4}{*}{$\operatorname{Ir} 90 \%$} & 0 & $0.39 \pm 0.01 \mathrm{~d}$ & $0.19 \pm 0.01 \mathrm{a}$ & $18.4 \pm 0.3 \mathrm{~d}$ & $4.21 \pm 0.08 \mathrm{~d}$ & $6.89 \pm 0.11 \mathrm{~d}$ & $122 \pm 3 \mathrm{e}$ & $41.3 \pm 0.8 \mathrm{e}$ \\
\hline & MGE & $0.39 \pm 0.01 \mathrm{~d}$ & $0.19 \pm 0.01 \mathrm{a}$ & $18.6 \pm 0.3 \mathrm{~d}$ & $4.23 \pm 0.08 \mathrm{~d}$ & $6.91 \pm 0.11 \mathrm{~d}$ & $426 \pm 10 \mathrm{~d}$ & $49.2 \pm 1.0 \mathrm{~d}$ \\
\hline & MGEPA $_{P A}$ & $0.40 \pm 0.01 \mathrm{~d}$ & $0.19 \pm 0.01 \mathrm{a}$ & $18.6 \pm 0.3 \mathrm{~d}$ & $4.25 \pm 0.08 \mathrm{~d}$ & $6.94 \pm 0.12 \mathrm{~d}$ & $548 \pm 13 c$ & $59.8 \pm 1.2 c$ \\
\hline & 0 & $0.48 \pm 0.01 c$ & $0.12 \pm 0.00 c$ & $29.4 \pm 0.5 c$ & $12.8 \pm 0.21 c$ & $10.4 \pm 0.18 c$ & $562 \pm 14 c$ & $61.4 \pm 1.4 c$ \\
\hline \multirow[t]{2}{*}{ Ir60\% } & MGE & $0.54 \pm 0.01 \mathrm{~b}$ & $0.15 \pm 0.01 \mathrm{~b}$ & $33.2 \pm 0.5 \mathrm{~b}$ & $15.6 \pm 0.29 b$ & $13.2 \pm 0.22 b$ & $798 \pm 17 b$ & $74.5 \pm 1.5 \mathrm{~b}$ \\
\hline & $\mathrm{MGE}_{\mathrm{PA}}$ & $0.63 \pm 0.02 \mathrm{a}$ & $0.19 \pm 0.01 \mathrm{a}$ & $38.8 \pm 0.6 \mathrm{a}$ & $19.2 \pm 0.37 \mathrm{a}$ & $17.6 \pm 0.31 \mathrm{a}$ & $994 \pm 21 \mathrm{a}$ & $98.6 \pm 1.9 \mathrm{a}$ \\
\hline \multirow{3}{*}{$\operatorname{Ir} 30 \%$} & 0 & $0.18 \pm 0.00 \mathrm{e}$ & $0.11 \pm 0.00 \mathrm{~d}$ & $12.2 \pm 0.2 \mathrm{e}$ & $2.31 \pm 0.04 \mathrm{e}$ & $5.04 \pm 0.10 \mathrm{e}$ & $101 \pm 2 \mathrm{f}$ & $31.0 \pm 0.5 \mathrm{f}$ \\
\hline & MGE & $0.18 \pm 0.00 \mathrm{e}$ & $0.11 \pm 0.00 \mathrm{~d}$ & $12.3 \pm 0.2 \mathrm{e}$ & $2.33 \pm 0.04 \mathrm{e}$ & $5.02 \pm 0.09 \mathrm{e}$ & $104 \pm 2 \mathrm{f}$ & $31.0 \pm 0.5 \mathrm{f}$ \\
\hline & $\mathrm{MGE}_{\mathrm{PA}}$ & $0.19 \pm 0.00 \mathrm{e}$ & $0.11 \pm 0.00 \mathrm{~d}$ & $12.3 \pm 0.2 \mathrm{e}$ & $2.34 \pm 0.05 \mathrm{e}$ & $5.05 \pm 0.10 \mathrm{e}$ & $107 \pm 2 \mathrm{f}$ & $31.4 \pm 0.6 \mathrm{f}$ \\
\hline \multicolumn{9}{|c|}{$\begin{array}{c}\text { Two way ANOVA } \\
\text { results }\end{array}$} \\
\hline \multicolumn{2}{|c|}{ Drought (D) } & $(*)$ & $(*)$ & $(*)$ & $(*)$ & $\left({ }^{*}\right)$ & $(* *)$ & $\left(^{*}\right)$ \\
\hline \multicolumn{2}{|c|}{ MGE } & $(*)$ & $(*)$ & $(*)$ & $(*)$ & $(*)$ & $(* *)$ & $(* *)$ \\
\hline \multicolumn{2}{|c|}{$\mathrm{D} \times \mathrm{MGE}$} & $(*)$ & $(*)$ & $(*)$ & $(*)$ & (NS) & $(* *)$ & $(*)$ \\
\hline
\end{tabular}

Values presented within columns are means $(n=5) \pm$ SE. The experiment was repeated three times. Means followed by different letter are significantly different $(p \leq 0.05) .\left(^{* *}\right)$ or $\left(^{*}\right)$ significant at 1 or $5 \%$ probability levels, respectively, and (NS) means not significant.

\subsection{Accumulation of Polyamines (PAs)}

The accumulation of PAs (PUT, SPD, and SPM) in wheat plants was markedly raised under Ir60\% treatment, while it reduced considerably under Ir30\% treatment compared to Ir90\% treatment (Table 7). Under optimal irrigation, PAs accumulation was significantly increased with seed soaking in MGE or MGE PA, with higher efficacy of MGE $_{\mathbf{P A}}$ than MGE compared to the corresponding control. MGE or MGE PA failed to increase PAs accumulation under Ir30\%. However, under Ir60\%, MGE increasing accumulation of all PAs compared to the corresponding control. The interaction (drought $\times$ MGE) was significant $(p \leq 0.05$ or 0.01$)$ for all PAs (Table 7). 
Table 7. Positive seed priming impacts using maize grain extract without (MGE, $2 \%$ ) or with polyamines enrichment $\left(\mathrm{MGE}_{\mathrm{PA}}, 2 \%\right.$ ) on polyamines; PAs (putrescine; PuT, spermidine; $\mathrm{SpD}$, and spermine; SpM) contents (nmol g $\left.{ }^{-1} \mathrm{FW}\right)$ of drought-stressed wheat plants.

\begin{tabular}{|c|c|c|c|c|}
\hline \multicolumn{2}{|c|}{ Treatments } & \multicolumn{3}{|c|}{ Parameters } \\
\hline Drought & MGE \% & PuT Content & SpD Content & SpM Content \\
\hline \multirow{4}{*}{$\operatorname{Ir} 90 \%$} & 0 & $10.3 \pm 0.2 \mathrm{e}$ & $59.8 \pm 0.7 \mathrm{e}$ & $54.2 \pm 0.6 \mathrm{e}$ \\
\hline & MGE & $15.2 \pm 0.2 \mathrm{~d}$ & $71.4 \pm 0.8 \mathrm{~d}$ & $64.1 \pm 0.6 \mathrm{~d}$ \\
\hline & MGE $_{P A}$ & $18.4 \pm 0.3 c$ & $78.2 \pm 0.8 c$ & $72.8 \pm 0.7 c$ \\
\hline & 0 & $18.5 \pm 0.3 c$ & $77.8 \pm 0.7 c$ & $70.9 \pm 0.7 c$ \\
\hline \multirow[t]{2}{*}{$\operatorname{Ir} 60 \%$} & MGE & $22.2 \pm 0.3 b$ & $86.1 \pm 0.9 b$ & $79.4 \pm 0.8 b$ \\
\hline & MGE $_{P A}$ & $25.6 \pm 0.4 \mathrm{a}$ & $94.4 \pm 0.9 \mathrm{a}$ & $87.5 \pm 0.8 \mathrm{a}$ \\
\hline \multirow{3}{*}{$\operatorname{Ir} 30 \%$} & 0 & $10.1 \pm 0.1 \mathrm{e}$ & $38.2 \pm 0.3 \mathrm{f}$ & $29.6 \pm 0.3 \mathrm{f}$ \\
\hline & MGE & $10.4 \pm 0.2 \mathrm{e}$ & $39.0 \pm 0.4 \mathrm{f}$ & $29.8 \pm 0.2 \mathrm{f}$ \\
\hline & MGE $_{\mathrm{PA}}$ & $10.4 \pm 0.2 \mathrm{e}$ & $39.4 \pm 0.4 \mathrm{f}$ & $30.4 \pm 0.3 \mathrm{f}$ \\
\hline \multicolumn{5}{|c|}{ Two-way ANOVA results } \\
\hline \multicolumn{2}{|c|}{ Drought (D) } & $(*)$ & $(*)$ & $(*)$ \\
\hline \multicolumn{2}{|c|}{ MGE } & $(*)$ & $(*)$ & $(*)$ \\
\hline \multicolumn{2}{|c|}{$\mathrm{D} \times \mathrm{MGE}$} & $(*)$ & $(*)$ & $(*)$ \\
\hline
\end{tabular}

Values presented within columns are means $(n=5) \pm$ SE. The experiment was repeated three times. Means followed by different letter are significantly different $(p \leq 0.05) .\left({ }^{*}\right)$ significant at $5 \%$ probability levels, respectively, and (NS) means not significant.

\subsection{Expression of Biosynthesis Genes of Polyamines (PAs)}

Ir60\% treatment up-regulated and increased the transcription of all biosynthesis genes of PAs (ADC, SPDS, SPMS, SAMDC, and DHS), but it did not affect ODC gene transcription compared to Ir $30 \%$ treatment, which did not affect gene transcription (Table 8). Seed

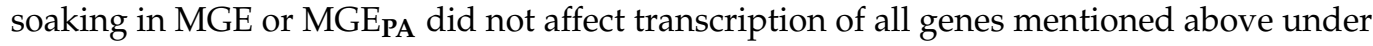
Ir $30 \%$. However, under either Ir $60 \%$ or Ir $90 \%$, all gene transcripts were up-regulated and in-

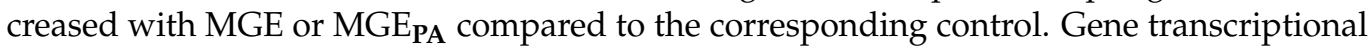
responses were more efficient under Ir60\% than Ir90\%, with higher efficacy for MGE $_{\mathbf{P A}}$ than MGE. The interaction (drought $\times$ MGE) was significant $(p \leq 0.05$ or 0.01 ) for all tested biosynthesis genes of PAs (Table 8).

Table 8. Positive seed priming impacts using maize grain extract without (MGE, $2 \%$ ) or with polyamines enrichment $\left(\mathrm{MGE}_{\mathrm{PA}}, 2 \%\right)$ on relative expression of PAs biosynthetic genes (by qPCR) of drought-stressed wheat plants.

\begin{tabular}{|c|c|c|c|c|c|c|c|}
\hline \multicolumn{2}{|c|}{ Treatments } & \multicolumn{6}{|c|}{ Parameters } \\
\hline Drought & MGE \% & ADC & ODC & SPDS & SPMS & SAMDC & DHS \\
\hline \multirow{4}{*}{$\operatorname{Ir} 90 \%$} & 0 & $1.0 \pm 0.02 \mathrm{e}$ & $1.00 \pm 0.01 \mathrm{a}$ & $1.0 \pm 0.01 \mathrm{f}$ & $1.00 \pm 0.02 \mathrm{e}$ & $1.0 \pm 0.01 \mathrm{e}$ & $1.0 \pm 0.01 \mathrm{f}$ \\
\hline & MGE & $1.4 \pm 0.03 \mathrm{~d}$ & $1.01 \pm 0.02 \mathrm{a}$ & $1.6 \pm 0.02 \mathrm{e}$ & $1.22 \pm 0.03 \mathrm{~d}$ & $2.1 \pm 0.03 \mathrm{~d}$ & $1.7 \pm 0.02 \mathrm{e}$ \\
\hline & $\mathrm{MGE}_{\mathrm{PA}}$ & $1.7 \pm 0.03 c$ & $1.01 \pm 0.01 \mathrm{a}$ & $1.9 \pm 0.02 \mathrm{~d}$ & $1.38 \pm 0.03 c$ & $2.5 \pm 0.05 c$ & $2.1 \pm 0.02 \mathrm{~d}$ \\
\hline & 0 & $1.5 \pm 0.02 \mathrm{~d}$ & $1.02 \pm 0.02 \mathrm{a}$ & $2.2 \pm 0.03 c$ & $1.42 \pm 0.04 c$ & $2.4 \pm 0.03 c$ & $2.5 \pm 0.03 c$ \\
\hline \multirow[t]{3}{*}{ Ir60\% } & MGE & $1.8 \pm 0.03 b$ & $1.02 \pm 0.02 \mathrm{a}$ & $2.9 \pm 0.04 b$ & $1.60 \pm 0.05 b$ & $3.1 \pm 0.05 b$ & $3.0 \pm 0.05 b$ \\
\hline & $\mathrm{MGE}_{\mathrm{PA}}$ & $2.0 \pm 0.04 \mathrm{a}$ & $1.02 \pm 0.02 \mathrm{a}$ & $3.5 \pm 0.05 \mathrm{a}$ & $1.75 \pm 0.05 \mathrm{a}$ & $3.6 \pm 0.06 \mathrm{a}$ & $3.4 \pm 0.05 a$ \\
\hline & 0 & $1.0 \pm 0.02 \mathrm{e}$ & $1.00 \pm 0.01 \mathrm{a}$ & $1.0 \pm 0.02 \mathrm{f}$ & $1.00 \pm 0.02 \mathrm{e}$ & $1.0 \pm 0.01 \mathrm{e}$ & $1.0 \pm 0.01 \mathrm{f}$ \\
\hline \multirow[t]{2}{*}{$\operatorname{Ir} 30 \%$} & MGE & $1.0 \pm 0.02 \mathrm{e}$ & $1.00 \pm 0.01 \mathrm{a}$ & $1.0 \pm 0.02 \mathrm{f}$ & $1.00 \pm 0.02 \mathrm{e}$ & $1.0 \pm 0.02 \mathrm{e}$ & $1.0 \pm 0.01 \mathrm{f}$ \\
\hline & MGE $_{P A}$ & $1.0 \pm 0.02 \mathrm{e}$ & $1.00 \pm 0.01 \mathrm{a}$ & $1.0 \pm 0.01 \mathrm{f}$ & $1.00 \pm 0.02 \mathrm{e}$ & $1.0 \pm 0.01 \mathrm{e}$ & $1.0 \pm 0.01 \mathrm{f}$ \\
\hline \multicolumn{8}{|c|}{ Two-way ANOVA results } \\
\hline \multicolumn{2}{|c|}{ Drought (D) } & $(*)$ & (NS) & $(*)$ & $\left({ }^{*}\right)$ & $(* *)$ & $(* *)$ \\
\hline \multicolumn{2}{|c|}{ MGE } & $\left({ }^{*}\right)$ & (NS) & $(*)$ & $\left({ }^{*}\right)$ & $(* *)$ & $(*)$ \\
\hline \multicolumn{2}{|c|}{$\mathrm{D} \times \mathrm{MGE}$} & $(*)$ & (NS) & $(*)$ & $\left({ }^{*}\right)$ & $(*)$ & $\left({ }^{*}\right)$ \\
\hline
\end{tabular}

Values presented within columns are means $(n=5) \pm$ SE. The experiment was repeated three times. Means followed by different letter are significantly different $(p \leq 0.05)$. $\left(^{* *}\right)$ or $\left(^{*}\right)$ significant at 1 or $5 \%$ probability levels, respectively, and (NS) means not significant. 


\section{Discussion}

No information is available on soaking wheat seeds in maize grain extract enriched with anti-stress stimulants such as polyamines [PAs $\left.\left(\mathrm{MGE}_{\mathbf{P A}}\right)\right]$ to alleviate the adverse impacts of drought stress. In this study, improvements in wheat plant growth and yield under the effects of drought stress were obtained through improvements in physiological and biochemical attributes due to seed priming in MGE or MGE PA $_{\mathbf{P A}}$ with the advantage of MGE $_{\text {PA }}$. Some articles reported on significant changes in antioxidant defense system including enzymatic and non-enzymatic antioxidants after exposing different plants to drought stress $[7,8,16,63]$ In the current study, this finding corresponds to the measured up-regulation of the ascorbate-glutathione (AsA-GSH) cycle, glyoxalase system, transand cis-zeatin, and PAs and their biosynthetic genes under drought stress in response to soaking wheat seeds in MGE or $\mathrm{MGE}_{\mathbf{P A}}$. In this regard, the results acquired with $\mathrm{MGE}_{\mathbf{P A}}$ exceeded those gained with MGE due to enrichment of MGE PA $_{\text {with }}$ PAs that increased the efficiency of the extract. Nevertheless, previous studies indicated the importance of MGE (the extract without enrichment with PAs) in mitigating the adverse effects of some stresses (salinity, cadmium, sandy state, and salinity+drought) in some crop plants [22,24-27], but this study reported a higher benefit of $\mathrm{MGE}_{\mathbf{P A}}$ than MGE.

In this study, drought stress in terms of deficit irrigation water (DIw) was detrimental to the growth and yield of wheat plants. Damage to wheat growth and yield due to Ir $30 \%$ (severe drought; $30 \%$ of soil relative water content; SRWC) was more severe than that from Ir60\% (moderate drought; 60\% of SRWC) (Figures 1 and 2, Table 9). The plants did not survive under severe drought even with pretreatment with both extracts (plants died during the flowering stage; Figure 1). This negative finding can be attributed to the drought affecting plant physiology and biochemistry (Figures 1 and 2, Tables 2-9). In this study, the decreased growth and yield of drought-stressed wheat plants associated with the overproduction of reactive species of oxygen (ROS), especially $\mathrm{H}_{2} \mathrm{O}_{2}$ and $\mathrm{O}_{2}{ }^{\bullet-}$ (Tables 2, 3 and 9). This finding is consistent with that obtained by Noctor et al. [64] and Wang et al. [65]. The plant's first response to drought is closed leaf stomata to reduce loss of water through transpiration (Tables 2,3 and 9). As a result of continuous photosynthesis in light, the depletion of intercellular $\mathrm{CO}_{2}$ concentration is facilitated by an increased gas diffusion barrier. Therefore, the low $\mathrm{CO}_{2}$ availability catalyzes the oxygenation of ribulose1,5-bisphosphate, thereby increasing the production of photorespiratory $\mathrm{H}_{2} \mathrm{O}_{2}$ in cell peroxisomes [64]. Drought treatments negatively affected leaf photosynthetic pigments, photosynthetic efficiency, gas exchange parameters, cell membranes (increase in lipid peroxidation; MDA and ionic leakage; EL, and decrease in stability index), RWC, zeatins, osmoprotectant and PAs contents, antioxidant enzyme, AsA-GSH cycle and glyoxalase system activities, and transcription of PAs biosynthetic genes (Figures 1 and 2, Tables 2-9). Drought-stimulated oxidative stress (increased production of $\mathrm{H}_{2} \mathrm{O}_{2}$ and $\mathrm{O}_{2}{ }^{\bullet-}$; Tables 2, 3 and 9) caused these negative findings. Over Ir $60 \%$, Ir $30 \%$ caused exacerbation of MDA and EL and severely affected PSII function efficiency (Figure 2 and Table 9), causing stomatal closure (Tables 2 and 9), which disrupted the plant antioxidant system (Tables 4-9), and eventually, the plant did not survive. 


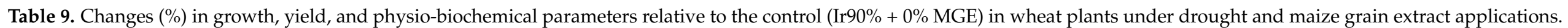

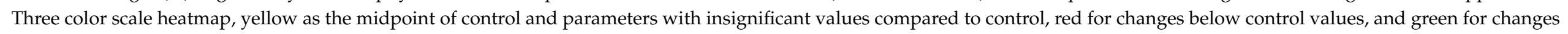
over control values.

\begin{tabular}{|c|c|c|c|c|c|c|c|c|c|}
\hline Parameters & Ir90\% (Control) & $\operatorname{Ir} 90 \%$ + MGE & Ir90\% + MGE & $\operatorname{Ir} 60 \%$ & $\begin{array}{c}\text { Treatments } \\
\text { Ir60\% + } \\
\text { MGE }\end{array}$ & $\operatorname{Ir} 60 \%+M G E_{P A}$ & Ir $30 \%$ & $\begin{array}{c}\text { Ir } 30 \%+ \\
\text { MGE }\end{array}$ & $\operatorname{Ir} 30 \%+M G E_{P A}$ \\
\hline Shoot FW & c & $+11.2 \mathrm{~b}$ & $+28.9 \mathrm{a}$ & $-21.8 \mathrm{e}$ & $-9.1 \mathrm{~d}$ & $+0.5 \mathrm{c}$ & $-48.7 \mathrm{f}$ & $-48.2 \mathrm{f}$ & $-48.2 \mathrm{f}$ \\
\hline Shoot DW & c & $+15.7 \mathrm{~b}$ & $+35.0 \mathrm{a}$ & $-34.5 \mathrm{e}$ & $-20.6 d$ & $-0.9 \mathrm{c}$ & $-72.6 \mathrm{f}$ & $-72.4 \mathrm{f}$ & $-72.6 \mathrm{f}$ \\
\hline Grain yield & c & $+10.0 \mathrm{~b}$ & $+21.3 \mathrm{a}$ & $-51.6 \mathrm{e}$ & $-36.7 d$ & $0.0 \mathrm{c}$ & PD * & PD & PD \\
\hline Grain weight & $\mathbf{a}$ & $+0.40 \mathrm{a}$ & $+1.6 \mathrm{a}$ & $-29.8 c$ & $-18.5 b$ & $0.0 \mathrm{a}$ & PD & PD & PD \\
\hline Chlorophylls & c & $+7.6 \mathrm{~b}$ & $+20.1 \mathrm{a}$ & $-27.2 \mathrm{e}$ & $-16.3 \mathrm{~d}$ & $+0.5 \mathrm{c}$ & $-53.3 \mathrm{f}$ & $-52.2 \mathrm{f}$ & $-52.2 \mathrm{f}$ \\
\hline Carotenoids & c & $+11.4 \mathrm{~b}$ & $+25.0 \mathrm{a}$ & $-18.2 \mathrm{e}$ & $-6.8 d$ & $+2.3 \mathrm{c}$ & $-36.4 \mathrm{f}$ & $-36.4 \mathrm{f}$ & $-34.1 \mathrm{f}$ \\
\hline PI & c & $+11.4 \mathrm{~b}$ & $+25.7 \mathrm{a}$ & $-11.4 \mathrm{e}$ & $-5.7 \mathrm{~d}$ & $0.0 \mathrm{c}$ & $-28.6 \mathrm{f}$ & $-27.1 \mathrm{f}$ & $-25.7 \mathrm{f}$ \\
\hline Hill activity & c & $+9.1 \mathrm{~b}$ & $+27.3 \mathrm{a}$ & $-18.2 \mathrm{e}$ & $-9.1 \mathrm{~d}$ & $0.0 \mathrm{c}$ & $-36.4 \mathrm{f}$ & $-36.4 \mathrm{f}$ & $-36.4 \mathrm{f}$ \\
\hline$P n$ & c & $+4.3 \mathrm{~b}$ & $+10.3 \mathrm{a}$ & $-18.1 \mathrm{e}$ & $-10.3 \mathrm{~d}$ & $0.0 \mathrm{c}$ & $-58.6 \mathrm{f}$ & $-58.6 \mathrm{f}$ & $-57.8 \mathrm{f}$ \\
\hline$A$ & b & $+6.9 \mathrm{a}$ & $+7.6 \mathrm{a}$ & $-22.8 d$ & $-13.1 c$ & $-0.7 \mathrm{~b}$ & $-53.8 \mathrm{e}$ & $-53.1 \mathrm{e}$ & $-53.1 \mathrm{e}$ \\
\hline$g s$ & c & $+7.6 \mathrm{~b}$ & $+19.3 \mathrm{a}$ & $-36.4 \mathrm{e}$ & $-20.2 \mathrm{~d}$ & $+0.3 \mathrm{c}$ & $-65.7 \mathrm{f}$ & $-65.1 \mathrm{f}$ & $-64.5 \mathrm{f}$ \\
\hline$E$ & c & $+11.1 \mathrm{~b}$ & $+27.8 \mathrm{a}$ & $-38.9 \mathrm{e}$ & $-22.2 \mathrm{~d}$ & $0.0 \mathrm{c}$ & $-72.2 \mathrm{f}$ & $-72.2 \mathrm{f}$ & $-72.2 \mathrm{f}$ \\
\hline $\mathrm{H}_{2} \mathrm{O}_{2}$ & d & $-0.6 \mathrm{~d}$ & $-0.6 \mathrm{~d}$ & $+54.3 \mathrm{~b}$ & $+32.4 \mathrm{c}$ & $-1.1 \mathrm{~d}$ & $+171.0 \mathrm{a}$ & $+170.2 \mathrm{a}$ & $+169.6 \mathrm{a}$ \\
\hline $\mathrm{O}_{2} \cdot-$ & d & $-0.8 \mathrm{~d}$ & $-0.8 \mathrm{~d}$ & $+109.9 \mathrm{~b}$ & $+47.9 \mathrm{c}$ & $-0.8 \mathrm{~d}$ & $+248.8 \mathrm{a}$ & $+247.1 \mathrm{a}$ & $+247.1 \mathrm{a}$ \\
\hline MDA & d & $-0.4 \mathrm{~d}$ & $-0.6 \mathrm{~d}$ & $+77.1 \mathrm{~b}$ & $+47.0 \mathrm{c}$ & $-0.5 \mathrm{~d}$ & $+216.5 \mathrm{a}$ & $+216.0 \mathrm{a}$ & $+215.8 \mathrm{a}$ \\
\hline MSI (\%) & $\mathbf{a}$ & $+0.8 \mathrm{a}$ & $+1.6 \mathrm{a}$ & $-27.8 c$ & $-8.7 b$ & $-0.3 \mathrm{a}$ & $-53.3 \mathrm{~d}$ & $-52.8 d$ & $-51.4 \mathrm{~d}$ \\
\hline RWC (\%) & $\mathbf{a}$ & $+0.7 \mathrm{a}$ & $+1.9 \mathrm{a}$ & $-28.8 \mathrm{c}$ & $-13.0 \mathrm{~b}$ & $-0.1 \mathrm{a}$ & $-53.5 \mathrm{~d}$ & $-53.1 \mathrm{~d}$ & $-52.3 \mathrm{~d}$ \\
\hline SOD & d & $0.0 \mathrm{~d}$ & $+0.2 \mathrm{~d}$ & $+37.4 \mathrm{c}$ & $+48.0 \mathrm{~b}$ & $+67.0 \mathrm{a}$ & $-35.2 \mathrm{e}$ & $-35.0 \mathrm{e}$ & $-35.2 \mathrm{e}$ \\
\hline CAT & d & $0.0 \mathrm{~d}$ & $0.0 \mathrm{~d}$ & $+34.6 c$ & $+56.5 \mathrm{~b}$ & $+79.4 \mathrm{a}$ & $-38.9 \mathrm{e}$ & $-38.9 \mathrm{e}$ & $-38.6 \mathrm{e}$ \\
\hline GST & d & $0.0 \mathrm{~d}$ & $+0.6 \mathrm{~d}$ & $+16.9 c$ & $+41.6 \mathrm{~b}$ & $+64.0 \mathrm{a}$ & $-33.7 \mathrm{e}$ & $-33.7 \mathrm{e}$ & $-33.1 \mathrm{e}$ \\
\hline AsA & $\mathbf{b}$ & $0.0 \mathrm{~b}$ & $+2.3 b$ & $-25.6 c$ & $0.0 \mathrm{~b}$ & $+11.6 \mathrm{a}$ & $-44.2 \mathrm{~d}$ & $-44.2 \mathrm{~d}$ & $-41.9 \mathrm{~d}$ \\
\hline APX & d & $0.0 \mathrm{~d}$ & $+1.1 \mathrm{~d}$ & $+67.4 \mathrm{c}$ & $+87.0 \mathrm{~b}$ & $+114.1 \mathrm{a}$ & $-46.7 \mathrm{e}$ & $-46.7 \mathrm{e}$ & $-45.7 \mathrm{e}$ \\
\hline GR & d & $0.0 \mathrm{~d}$ & $+1.5 \mathrm{~d}$ & $+25.4 \mathrm{c}$ & $+35.8 \mathrm{~b}$ & $+47.8 \mathrm{a}$ & $-47.8 \mathrm{e}$ & $-46.3 \mathrm{e}$ & $-46.3 \mathrm{e}$ \\
\hline MDHAR & $\mathbf{a}$ & $+0.3 \mathrm{a}$ & $+0.8 \mathrm{a}$ & $-47.1 \mathrm{c}$ & $-28.1 b$ & $-0.8 \mathrm{a}$ & $-62.6 \mathrm{~d}$ & $-62.6 \mathrm{~d}$ & $-62.5 d$ \\
\hline DHAR & $\mathbf{a}$ & $0.0 \mathrm{a}$ & $+0.8 \mathrm{a}$ & $-45.5 c$ & $-25.8 \mathrm{~b}$ & $-2.3 \mathrm{a}$ & $-62.1 \mathrm{~d}$ & $-61.4 \mathrm{~d}$ & $-60.6 d$ \\
\hline GSH & d & $0.0 \mathrm{~d}$ & $+1.1 \mathrm{~d}$ & +19.1 c & $+39.3 \mathrm{~b}$ & $+67.4 \mathrm{a}$ & $-28.1 \mathrm{e}$ & $-28.1 \mathrm{e}$ & $-25.8 \mathrm{e}$ \\
\hline GSSG & d & $0.0 \mathrm{~d}$ & $+0.9 \mathrm{~d}$ & $+85.6 \mathrm{c}$ & $+133.8 \mathrm{~b}$ & +186.0 a & $-35.6 \mathrm{e}$ & $-35.1 \mathrm{e}$ & $-34.2 \mathrm{e}$ \\
\hline Gly I & d & $0.0 \mathrm{~d}$ & $+2.6 \mathrm{~d}$ & $+23.1 \mathrm{c}$ & $+38.5 \mathrm{~b}$ & $+61.5 \mathrm{a}$ & $-53.8 \mathrm{e}$ & $-53.8 \mathrm{e}$ & $-51.3 \mathrm{e}$ \\
\hline Gly II & $\mathbf{a}$ & $0.0 \mathrm{a}$ & $0.0 \mathrm{a}$ & $-36.8 \mathrm{c}$ & $-21.1 \mathrm{~b}$ & $0.0 \mathrm{a}$ & $-42.1 \mathrm{~d}$ & $-42.1 \mathrm{~d}$ & $-42.1 \mathrm{~d}$ \\
\hline
\end{tabular}


Table 9. Cont.

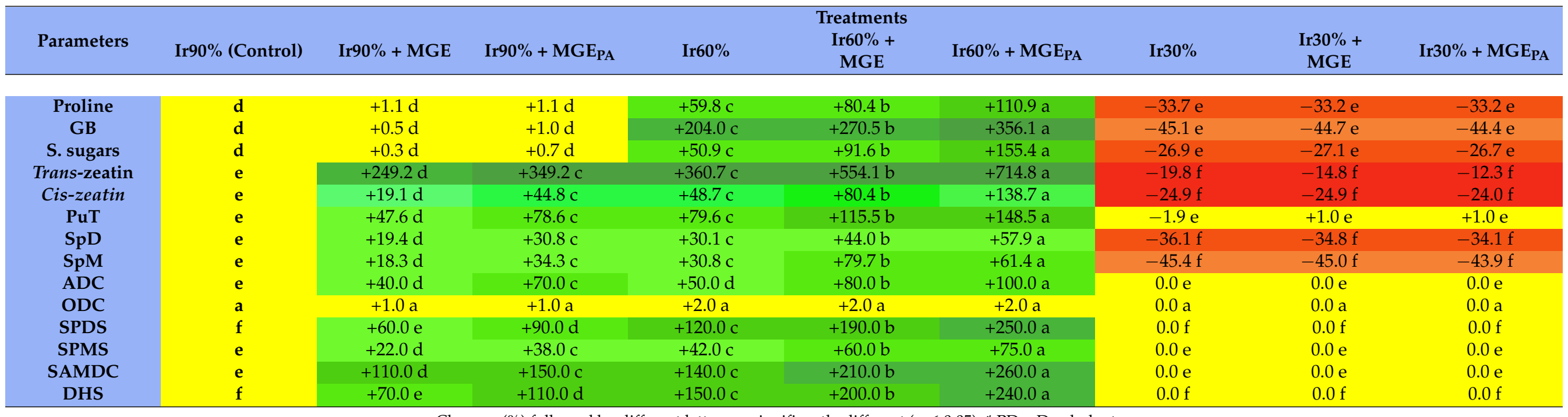

Changes (\%) followed by different letter are significantly different $(p \leq 0.05) .{ }^{*} \mathrm{PD}=$ Dead plants. 
However, the advantage of seed soaking in both extracts (MGE or MGE $\mathrm{PA}$ ) was reported under moderate drought (Ir60\%). The higher benefit of MGE be attributed to the additional benefits of PAs granted through pretreatment with MGE ${ }_{\mathbf{P A}}$, where PAs play important roles in mitigating the damage caused by different stresses on crop plants [29,66-68]. Yildiztugay et al. [69] reported a relationship between the plant's biomass (growth) and its water content. Similarly, our results displayed this relationship (Figures 1 and 2, Tables 2, 3 and 9). Despite the benefit of pretreatment with MGE, MGE $\mathbf{P A}$ enabled wheat plants to function normally under Ir60\% and revealed growth (biomass) and leaf relative water content (RWC), and thus yield similar to that gained by the optimum irrigated plants (Ir90\%; control). Wheat plants pretreated with MGE $_{\mathbf{P A}}$ and subjected to Ir60\% had leaf photosynthetic pigments, photosynthesis efficiency (Fv/Fm, PI, and hill reaction activity; HRA), and gas exchange parameters identical to those obtained with well-watered plants (Figures 1 and 2, Tables 2, 3 and 9). The damage caused by Ir60\% was repaired with $\mathrm{MGE}_{\mathbf{P A}}$. During the soaking process, the seeds can absorb the bioactive ingredients from the extract such as antioxidants (AsA and GSH) and hormones (salicylic acid and cytokinins (CKs), especially $t-Z$ and $c-Z$ ), in addition to the extract PAs (Table 1). These bioactive ingredients enrich the seeds and accelerate metabolism, giving them robust germination and strong seedlings to withstand drought stress.

Under Ir60\%, MGE PA preserved leaf photosynthetic pigments and photosynthetic efficiency along with gas exchange parameters, which contributed to the maintenance of wheat yield (Figures 1 and 2, Tables 2, 3 and 9). In this regard, Farooq et al. [16] reported a positive correlation between chlorophyll contents and grain yield in wheat plants. Moreover, the maintenance of PSII function (HRA), antioxidant system, and biosynthesis genes of PAs (Tables 4-9) along with the beneficial impacts of the bioactive stimulants present in MGE $_{\mathbf{P A}}$ (Table 1) contributed well to the plant's performance under drought

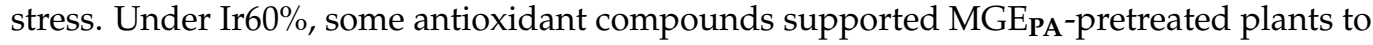
cause cell membrane stabilization by suppressing $\mathrm{H}_{2} \mathrm{O}_{2}$ and $\mathrm{O}_{2}{ }^{\bullet-}$ levels, thus reducing MDA and EL levels (Tables 3 and 9). In this study, as drought stress caused a reduction in chlorophyll content, it caused leaf yellowing and senescence (data not shown). This finding can be attributed to a degradation of chlorophyll and a dysregulation of the photosynthetic apparatus, including decreased HRA, and thus a reduction in wheat yield. Nevertheless, MGE PA enabled wheat plants to stay green by maintaining the chlorophyll content for as long as possible, thus producing better under drought stress (Figure 2, Table 9) due to the bioactive stimulants present in MGEPA. This finding is consistent with that obtained by [22,24-27] under different abiotic stress conditions. Drought-stressed wheat plant becomes better performer (in terms of growth and yield characteristics) when pretreated with MGE, which has a high ROS-scavenging activity (88.6 $\pm 1.6 \%$ ) due to the high contents of different antioxidants, plant hormones, and PAs present in MGE (Table 1). The performance of stressed plant becomes higher with MGE $\mathbf{P A}$ due to the PAs added to MGE. Ghassemi et al. [70] reported that PAs improved root growth and development and adjusted cellular water potential after emergence, leading to increased nutrient uptake and translocation in drought-stressed plants. In addition, the enhanced translocation of assimilates, which are photosynthesized in plant leaves, to the edible portion (the grains) is attributed to the longer duration of photosynthesis due to the green leaf survival merit, resulting in a longer period of grain filling under stress [16]. This finding is in line with the finding of this study, which is likely due to the ability of MGEPA-pretreated wheat plants to stimulate the activity of the antioxidant system to withstand DIw stress (Tables 4-9) and enable the plants to perform better with regard to the meristematic tissue activities, including the stimulation of cell division and expansion due to maintaining adequate water (Figures 1 and 2, Tables 2, 3 and 9). As a result, it becomes possible to obtain a satisfactory improvement in the growth of drought-stressed plants due to the richness of MGE, especially after its enrichment with PAs, in phytohormones, auxins, CKs including zeatins, gibberellins (GAs), and PAs (Table 1). Therefore, hormonal homeostasis could be induced with MGE $_{\mathbf{P A}}$ as a potential mechanism for boosting DIw stress tolerance in plants. 
This hormonal homeostasis mechanism could function through a complex cross-talk among auxins, CKs and GAs, as well as among plant hormones, PAs, and other biostimulants as an anti-stress network in favor of the plant's response to DIw stress.

In this study, under low availability of water, MGE $_{\mathbf{P A}}$ pretreatment replaced the detrimental effects of DIw to marked increases in wheat growth and yield due to the increase in gas exchange parameters, photosynthetic efficiency including HRA, and the biosynthesis of chlorophyll catalyzed by the biostimulants present in MGE $\mathbf{P A}$, which contributed to elimination of the biomarkers of oxidative stress $\left(\mathrm{H}_{2} \mathrm{O}_{2}\right.$ and $\left.\mathrm{O}_{2}{ }^{\bullet-}\right)$ and preservation of cell membranes and cell water content due to reduced levels of EL and MDA accumulation (Figures 1 and 2, Tables 2, 3 and 9). Different stresses cause a detrimental effect on EL and membrane lipids due to overproduced ROS, which disrupt chlorophyll biosynthesis due to disruption of the pigment protein complex functioning, de novo protein biosynthesis, and chlorophyll-associated components $[8,16,71,72]$. However, MGE PA $_{\text {bioactive promoters }}$ eliminated these harmful effects and mediated improvement in chlorophyll biosynthesis (Figures 1 and 2, Tables 2, 3 and 9). MGE PA pretreatment can stimulate the accumulation of different plant hormones in germinated seeds and thus seedlings, especially CKs, to improve the biosynthesis of chlorophylls as shown in Cortleven et al. [73]. In this study, MGE $_{\text {PA }}$ pretreatment improved photosynthesis efficiency and PSII activity (Fv/Fm, PI, and HRA), which could be attributed to suppressing the formation of $\operatorname{ROS}\left({ }^{1} \mathrm{O}_{2}, \mathrm{H}_{2} \mathrm{O}_{2}\right.$, and $\left.\mathrm{O}^{2 \bullet-}\right)$, thus protecting the chloroplast structure from DIw-induced oxidative stress damage (Figures 1 and 2, Tables 2, 3 and 9). In this regard, Tikkanen et al. [74] attributed a similar result to the safeguarded light reaction functioning in photosynthesis process, which is functionally synchronized with PSI and PSII enzymes. The functioning of both photosystems is connected to the generation of trans-thylakoid proton, which creates a $\mathrm{pH}$ difference for regulating the transfer of electrons to PSI under direct monitoring of the PGR5 protein. As a result of its richness in bioactive stimuli, especially plant hor-

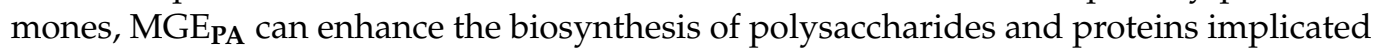
in photosynthesis regulation, in addition to mediating the synthesis of components of redox that may have contributed to photo-protection. In addition, MGE $\mathbf{P A}$ can increase carotenoids synthesis that can protect the photosynthesis system from the overproduction of ROS by up-regulating pigment-synthesizing enzyme activities associated with reducing the degradation of enzymes [25,26,75]. This result is consistent with Aldesuquy [76], who reported a positive synergistic impact of plant hormones on pigment and fine chloroplast structure in the flag leaf of stressed wheat plant.

As previously obtained in some works [22,24-27], reduced levels of $\mathrm{H}_{2} \mathrm{O}_{2}$ and $\mathrm{O}_{2}{ }^{\bullet-}$ and thus reduced lipid peroxidation and EL were obtained with the MGEPA-pretreated plant under DIw stress (Tables 3 and 9). The MGE-mediated enhanced membrane integrity (due to reduced membrane EL and MDA) could be attributed to the bioactive components in present MGE $\mathbf{P A}$ (Table 1), which contributed to the maintenance of the components of the antioxidant system, in addition to the low peroxidation levels, which were considerably influenced by DIw stress. MGE PA pretreatment considerably enhanced the activity of antioxidant enzymes and raised the contents of AsA and glutathione forms (e.g., GSH and GSSG), thus protecting the wheat plants from the oxidative stress $\left(\mathrm{H}_{2} \mathrm{O}_{2}\right.$ and $\left.\mathrm{O}_{2}{ }^{--}\right)$ stimulated by DIw. Similar results are previously gained [22,24-27] under different stresses. Integrally, after dismutating $\mathrm{O}_{2}{ }^{\bullet-}$ to $\mathrm{H}_{2} \mathrm{O}_{2}$ by SOD, APX and CAT convert $\mathrm{H}_{2} \mathrm{O}_{2}$ to $\mathrm{H}_{2} \mathrm{O}$ and $\mathrm{O}_{2}$. This mechanism reduces the formation of hydroxyl; $\mathrm{OH}^{-}$radicals [77]. Since MGE $_{\mathbf{P A}}$ is a rich source of bioactive stimulants, it may stimulate SOD up-regulation to further dismutate $\mathrm{O}_{2}{ }^{\bullet-}$ to $\mathrm{H}_{2} \mathrm{O}_{2}$. Plants pretreated with MGE PA possessed stimulated AsA and GSH accumulations, which could protect stressed plants from ROS damage stimulated by DIw. All antioxidants (GR, APX, MDHAR, DHAR, GSH, and AsA) are components of the ROS-scavenging pathway (the AsA-GSH cycle), which can be stimulated and upregulated by $\mathrm{MGE}_{\mathbf{P A}}$ to elevate tolerance strategies against any potential damage from oxidative stress in the wheat plant (Tables 6-9). This robust enhancement in the antioxidant system in the DIw-stressed wheat plants pretreated with MGE $_{\mathbf{P A}}$ resulted in a marked 
decrease in ROS accumulation (e.g., $\mathrm{H}_{2} \mathrm{O}_{2}$ and $\mathrm{O}_{2}{ }^{\bullet-}$ ) through the AsA-GSH cycle and thus increased the protection of pathways of photosynthesis, resulting in better performance (e.g., growth and yield productivity) of wheat plants (Tables 6-9). The AsA-GSH cycle includes a sequence of redox reactions, which includes bioactive participation of NADPH, GSH, and AsA [78]. Through this cycle, $\mathrm{H}_{2} \mathrm{O}_{2}$ molecules in cell cytosol and chloroplasts are scavenged by APX and CAT, thus preventing $\mathrm{H}_{2} \mathrm{O}_{2}$ diffusion to other organelles to avoid any damage. The improved functionality of the ASA-GSH cycle pathway due to pretreatment with MGE $_{\mathbf{P A}}$ effectively maintained redox components, including GSH and AsA. These redox components reduced the effects of oxidative stress $\left(\mathrm{H}_{2} \mathrm{O}_{2}\right.$ and $\mathrm{O}_{2}{ }^{\bullet-}$ ) stimulated by DIw. In this study, the increased antioxidant system activity was accompanied by an enhanced tolerance to DIw stress in the wheat plant. Similar results were gained by $[16,25,79]$. DHAR and MDHAR activities, which may be up-regulated after pretreatment with MGE $_{\mathbf{P A}}$, improved AsA and GSH to integrate the functions of other enzymes such as GR and APX with non-enzymatic antioxidant components. As a result, a comprehensive impact occurred on $\mathrm{H}_{2} \mathrm{O}_{2}$ neutralization and the availability of NADP to effectively protect the electron transport chain [80]. In this study, pretreatment with MGE $_{\mathbf{P A}}$ bioactive stimulants protected the electron transport chain implicated in photosynthesis, which may be attributed to the up-regulation of the $\mathrm{NADP}^{+} / \mathrm{NADPH}$ ratio. This mechanism prevents the flow of electrons into $\mathrm{O}_{2}$ to restrict $\mathrm{O}_{2}{ }^{\bullet-}$ production [81]. This study provides a preventive role of $\mathrm{MGE}_{\mathrm{PA}}$, given its richness in bioactive stimuli including plant hormones, for the photosynthesis system through the up-regulation of the antioxidant system components.

High levels of GSH can contribute significantly to $\mathrm{MGE}_{\mathbf{P A}}$-pretreated wheat plants to maintain the glycoxylase system for methylglyoxal elimination, which may reduce the chances of any dangerous genotoxic influence [82]. Glyoxylase I and II (Gly I and Gly II) constitute the main enzymatic components of the 'glyoxylase system'. MGE stimuli-stimulated up-regulation in the enzymatic activities of glyoxylase system could be associated with increased levels of GSH. This may have exploited the improved influences of methylglyoxal such as crosstalk with important signalling molecules such as $\mathrm{Ca}^{2+}, \mathrm{ABA}$,

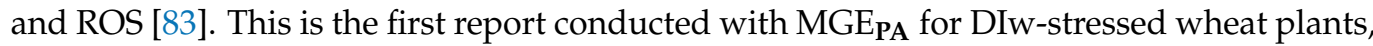

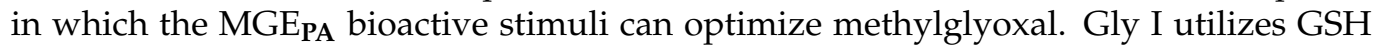
as a co-factor to convert the methylglyoxal to $S$-D-lactoylglutathione. In addition, Gly II produces GSH to contribute to redox homeostasis and protection against toxic species [84]. It can be concluded that improvement in endogenous GSH by pretreatment using MGE $_{\mathbf{P A}}$ bioactive stimuli and the functioning of methylglyoxal scavenging system could enhance stress tolerance in wheat plants. Ahanger et al. [85] explained up-regulation and the improvement of glyoxylase system activity due to exogenous treatment with kinetin. This result is consistent with our results through the application of CKs-containing MGE PA, $_{\text {, }}$ which improved activity of glyoxylase system (Tables 6 and 9). This enhanced glycosylase system activity may protect the system of electron transport by inhibiting any damage to chloroplasts and mitochondrial ultra-structures [82].

In this study, DIw encouraged osmoprotectant accumulations (e.g., GB, S. sugars, and proline), and these accumulations were further enhanced due to pretreatment with MGE $_{P A}$ bioactive stimulants. This accumulation of osmoprotectants may occur due to their absorption from $\mathrm{MGE}_{\mathbf{P A}}$ and/or their increased biosynthesis catalyzed by $\mathrm{MGE}_{\mathbf{P A}}$ in soaked seeds then seedlings. These increased osmoprotectants may give the seeds the driving force for strong germination and thus vigorous seedling growth under stress. Improvements of these osmolytes may be effective mechanisms to increase plant tolerance to DIw stress to maintain adequate cell water for healthy metabolism (Tables 2-9). The up-regulation of proline-synthesizing enzymes along with the down-regulation of proline-catabolizing enzymes and/or low incorporation into proteins leads to proline accumulation [86,87]. The improvement in GB, S. sugars, and proline accumulations as a result of $\mathrm{MGE}_{\mathbf{P A}}$ pretreatment resulted in the maintenance of water balance in DIw-stressed plants to help them withstand DIw stress and avoid increased EL and MDA in plant tissues for healthy 
metabolic processes, protection of protein turnover, enzymatic activities, and expression of stress protective proteins. These results indicate the protective role of MGE $\mathrm{PA}_{\mathbf{P A}}$ for healthy metabolic pathways and effective osmoregulation. Similar results were obtained by Ahanger and Agarwal [85] and Thakur and Sharma [88].

Bypassing MGE, MGE ${ }_{\mathrm{PA}}$ attenuated DIw stress and helped the photosynthetic system to perform efficiently (Figure 2, Table 9) in favor of plant metabolism [27], resulting in a lot of savings including hormonal contents (e.g., CKs such as $t-Z$ and $c-Z$ ) in DIw-stressed plants (Tables 6 and 9). Therefore, zeatins play a fundamental role in wheat plant response to DIw stress. Mediating plant response to stress, specifically CKs and their signaling components, predominantly regulate plant defense reactions [89]. T-Z and $c-Z$ have been reported to modulate the wheat plant's defense response to stress through several mechanisms including defense genes and hormonal regulation [26]. This result confirms the

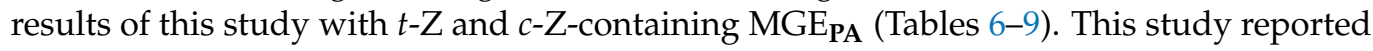
significant increases in trans-zeatin and cis-zeatin contents with MGE $_{\mathbf{P A}}$ pretreatment and supported wheat plant's tolerance to DIw stress (Figures 1 and 2, Tables 2-9). Previous reports indicated increased $t-Z$ activity compared to cis-zeatin, which was demonstrated in this study with a higher trans-zeatin content compared to $c-Z$, which may lead to an increase in the physiological role of $t-Z$ to stimulate a higher increase in PAs contents and their gene expressions under DIw stress due to pretreatment of the plant with MGE PA $_{\mathbf{P}}$ (Tables 6-9). This finding may be attributed to the transport, degradation, and conjugation processes of these hormones [90,91].

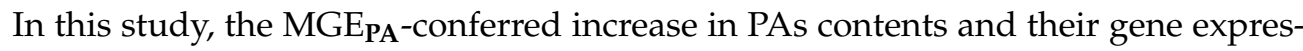
sions contributed to distinct defense mechanisms, which improved the tolerance of the wheat plant to DIw stress (Figures 1 and 2, Tables 2-9). The integrated defense mechanisms of antioxidant and osmoregulation systems along with hormonal content and gene

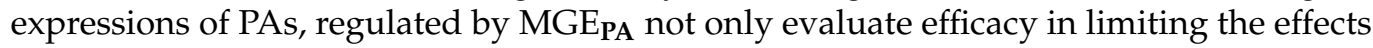
of hazardous stress but also modulate physiological status to terminate the trade-off of plant integrity related to defense responses (Figures 1 and 2, Tables 2-9). Therefore, wheat plants could survive better with MGE $_{P A}$ pretreatment in areas with a shortage of water such as dry ones. MGE ${ }_{\text {PA }}$ pretreatment stimulated more accumulation in PAs (PUT, SPD, and SPM) that act as signaling molecules to focus primarily on metabolism in relation to conservation against DIw stress in wheat plants (Tables 7 and 9). This finding, together with other cumulative zeatins and antioxidants (Tables 2-9), is likely due to the attenuation of the adverse effects of DIw stress through its powerful antioxidant roles $[29,67]$ and their gene expressions [26]. Cumulative levels of endogenous PAs under DIw stress conditions have been linked to effective up-regulation of SPDS, ADC, DHS, SPMS, and SAMDC gene expression but not to the ODC gene (Tables 8 and 9). Similar results were reported by Ebeed et al. [67] and Alharby et al. [26]. The results of this study indicate that PAs, PUT, SPD, and SPM were synthesized under DIw stress through the SPDS, ADC, DHS, SPMS, and SAMDC pathways (and not from ODC) in wheat plants. Various enzymes were involved in the pathway from PUT to SPM and SPD, including SAMDC, SPDS, and SPMS gene expression. The endogenous levels of SPD, SPM, and PUT that accumulated by MGE PA $_{\text {in }}$ DIw-stressed wheat plants were connected to the up-regulated gene expression levels of the ADC, SPDS, SPMS, SAMDC, and DHS genes, giving wheat plants a robust antioxidant defense to withstand DIw stress (Figures 1 and 2, Tables 2-9).

MGE $_{P A}$ efficiently outperformed MGE in promoting wheat plant growth, grain yield, physio-biochemistry, and antioxidant defense system components (Figures 1 and 2, Tables 2-9). These promoted results can be attributed to MGE $\mathbf{P A}^{\prime}$ 's further enrichment of PAs along with its high antioxidants and phytohormone contents, as well as its higher antioxidant activity (88.6\%) (Table 1). This makes MGE PA $_{\text {p }}$ possess pivotal mechanisms for inhibiting or at least minimizing the dangerous impacts of oxidative stress, cell membrane lipid peroxidation, and ionic leakage [25,26]. Therefore, MGE $_{\mathbf{P A}}$, an eco-friendly strategy, is a powerful natural extract that contains many antioxidants and biostimulants for plant growth under stress. 


\title{
5. Conclusions
}

Drought stress led to a severe decrease in wheat plant growth and yield due to stress negative effects such as decreased chlorophyll content, photosynthetic efficiency, water content, and gas exchange, in addition to PAs gene expression, and increased membrane lipid peroxidation and electrolyte leakage due to increased oxidative stress. These negative effects can be attenuated by using a plant extract such as biostimulants-rich MGE enriched with PAs (MGE $\mathbf{P A})$ as a seed soaking strategy. MGE $_{\mathbf{P A}}(2 \%)$ pretreatment promoted wheat plant growth and yield by improving photosynthetic efficiency, antioxidant and osmoregulation systems, and PAs gene expression. This was due to reduced oxidative stress damage by MGE $\mathbf{P A}$ through improved enzymatic and non-enzymatic antioxidant activities and up-regulation of the ascorbate-glutathione cycle and the glyoxalase system. All these positive results were reflected in the decrease in memebrane lipid peroxidation and the leakage of electrolytes under drought stress. This study predicts that plant extracts such as biostimulants-rich MGE enriched with PAs possess signaling networks that interfere with many physiological and biochemical pathways to develop DIw-tolerant crops for effective sustainable agriculture.

Author Contributions: H.F.A., K.R.H. and M.M.R. visualized and designed experiments, H.F.A., M.M.R. and Y.M.A. performed experiments, H.S.A.-Z., M.M.R. and H.F.A. analyzed data, H.A. and M.M.R. contributed to reagents/materials/analysis tools, H.F.A., H.S.A.-Z., K.R.H., Y.M.A. and H.A. wrote the manuscript, M.M.R. revised the manuscript. All authors have read and agreed to the published version of the manuscript.

Institutional Review Board Statement: Not applicable.

Informed Consent Statement: Not applicable.

Data Availability Statement: The data presented in this study are available upon request from the corresponding author.

Acknowledgments: This Project was funded by the Deanship of Scientific Research (DSR) at King Abdulaziz University, Jeddah, under grant no. (RG-1-130-40). The authors, therefore, acknowledge with thanks DSR for technical and financial support.

Conflicts of Interest: The authors declare that they have no conflict of interest.

\begin{abstract}
Abbreviations
MGE; Maize grain extract, $\mathrm{MGE}_{\mathbf{P A}}$; Maize grain extract enriched with polyamines, $\mathrm{O}_{2}{ }^{\bullet-}$; Superoxide, $\mathrm{H}_{2} \mathrm{O}_{2}$; Hydrogen peroxide, DIw; Deficit irrigation water, $\mathrm{CO}_{2}$; Carbon dioxide, ROS; reactive oxygen species, DNA; deoxyribonucleic acid, RNA; Ribonucleic acid, SOD; Superoxide dismutase, POD; Peroxidase, CAT; Catalase, GR; Glutathione reductase, APX; Ascorbate peroxidase, GST; Glutathione S-transferase, MDHAR; Monodehydroascorbate reductase, DHAR; Dehydroascorbate reductase, GSH; Reduced glutathione, GSSG; Oxidized glutathione, AsA; Ascorbate, OH; Hydroxyl, PAs; Polyamines, $\mathrm{mM}$; Millimole, $\mu \mathrm{M}$; Micromole, DAS; Days after sowing, SRWC; Soil relative water content, DPPH; 1,1-diphenyl-2-picrylhydrazyl, PUT; Putrescine, SPM, Spermine, SPD, Spermidine, HPLC; High-performance liquid chromatography, GC/MS; Gas chromatography-mass spectrometry, FW; Fresh weight, DW; Dry weight, PSII; Photosystem II, PI; performance index, Fv /Fm; A normalized ratio created by dividing variable fluorescence $(\mathrm{Fv})$ by maximum fluorescence $(\mathrm{Fm})$, $\mathrm{Pn}$; Net photosynthesis rate, $A ; \mathrm{CO}_{2}$ assimilation rate, $g s$; Stomatal conductance, E; Transpiration rate, MDA; Malondialdehyde, PVP; Polyvinyl pyrrolidone, NBT; 4-Nitro blue tetrazolium chloride, EDTA; Ethylenediamine tetraacetic acid, $\mathrm{HPO}_{3}$; Metaphosphoric acid, Gly I; Glyoxalase I, Gly II; Glyoxalase II, GB; Glycinebetaine, S. sugars; Soluble sugars, $\mathrm{H}_{2} \mathrm{SO}_{4}$; Sulphuric acid, nm; Nanometer, qRT-PCR; Real-Time Quantitative Reverse Transcription, nM; Nanometer, GAPDH; Glyceraldehyde 3phosphate dehydrogenase gene, ADC; Arginine decarboxylase gene, ODC; Ornithine decarboxylase gene, SAMDC; S-Adenosylmethionine decarboxylase proenzyme gene, SPDS; Spermidine synthase gene, SPMS, Spermine synthase gene, DHS; Deoxyhypusine synthase gene, GAPDH; Glyceraldehyde3-phosphate dehydrogenase gene, $t$-Z; Trans-zeatin-type cytokinin, $c$-Z; Cis-zeatin-type cytokinin, ANOVA; Analysis of variance, Ir60\%; Moderate drought, Ir30\%; Severe drought, Ir $90 \%$; Optimum
\end{abstract}


irrigation, HRA; Hill reaction activity, MSI; Membrane stability index, RWC; Relative water content, EL; Electrolyte leakage, CKs; Cytokinines, PGR5; Proton gradient regulation-5, AsA-GSH cycle; Ascorbate-glutathione cycle, and ABA; Abscisic acid.

\section{References}

1. Kiliç, H.; Yağbasanlar, T. The effect of drought stress on grain yield, yield components and some quality traits of durum wheat (Triticum turgidum ssp. durum) cultivars. Not. Bot. Hort Agrobot. Cluj-Napoca 2010, 38, 164-170.

2. Hammad, S.A.R.; Ali, O.A.M. Physiological and biochemical studies on drought tolerance of wheat plants by application of amino acids and yeast extract. Ann. Agric. Sci. 2014, 59, 133-145. [CrossRef]

3. Mahajan, S.; Tuteja, N. Cold, salinity and drought stresses: An overview. Arch. Biochem. Biophys. 2005, 444, 139-158. [CrossRef] [PubMed]

4. Abd El-Mageed, T.A.; Semida, W.M.; Rady, M.M. Moringa leaf extract as biostimulant improves water use efficiency, physiobiochemical attributes of squash plants under deficit irrigation. Agric. Water Manag. 2017, 193, 46-54. [CrossRef]

5. Abd El-Mageed, T.A.; Semida, W.M.; Taha, R.S.; Rady, M.M. Effect of summer-fall deficit irrigation on morpho-physiological, anatomical responses, fruit yield and water use efficiency of cucumber under salt affected soil. Sci. Hortic. 2018, 237, 148-155. [CrossRef]

6. Merwad, A.M.A.; Desoky, E.M.; Rady, M.M. Response of water deficit-stressed Vigna unguiculata performances to silicon, proline or methionine foliar application. Sci. Hortic. 2018, 228, 132-144. [CrossRef]

7. Desoky, E.M.; Mansour, E.; Yasin, M.A.T.; El-Sobky, E.E.A.; Rady, M.M. Improvement of drought tolerance in five different cultivars of Vicia faba with foliar application of ascorbic acid or silicon. Spanish J. Agric. Res. 2020, 18, e0802. [CrossRef]

8. Rady, M.M.; Belal, H.E.E.; Gadallah, F.M.; Semida, W.M. Selenium application in two methods promotes drought tolerance in Solanum lycopersicum plant by inducing the antioxidant defense system. Sci. Hortic. 2020, 266, 109290. [CrossRef]

9. Parvaiz, A.; Satyawati, S. Salt stress and phyto-biochemical responses of plants-a review. Plant Soil Environ. 2008, 54, 89-99. [CrossRef]

10. Tariq, A.; Pan, K.; Olatunji, O.A.; Graciano, C.; Li, Z.; Sun, F.; Sun, X.; Song, D.; Chen, W.; Zhang, A.; et al. Phosphorous application improves drought tolerance of Phoebe zhennan. Front. Plant Sci. 2017, 8, 1561. [CrossRef]

11. Apel, K.; Hirt, H. Reactive oxygen species: Metabolism, oxidative stress, and signal transduction. Ann. Rev. Plant Biol. 2004, 55, 373-399. [CrossRef]

12. Shigeoka, S.; Ishikawa, T.; Tamoi, M.; Miyagawa, Y.; Takeda, T.; Yabuta, Y.; Yoshimura, K. Regulation and function of ascorbate peroxidase isoenzymes. J. Exp. Bot. 2002, 53, 1305-1319. [CrossRef]

13. Tariq, A.; Pan, K.; Olatunji, O.A.; Graciano, C.; Li, Z.; Sun, F.; Zhang, L.; Wu, X.; Chen, W.; Song, D.; et al. Phosphorous fertilization alleviates drought effects on Alnus cremastogyne by regulating its antioxidant and osmotic potential. Sci. Rep. 2018, 8, 5644. [CrossRef]

14. Sanoubar, R.; Cellini, A.; Gianfranco, G.; Spinelli, F. Osmoprotectants and antioxidative enzymes as screening tools for salinity tolerance in radish (Raphanus sativus). Hortic. Plant J. 2020, 6, 14-24. [CrossRef]

15. Demirevska, K.; Zasheva, D.; Dimitrov, R.; Simova-Stoilova, L.; Stamenova, M.; Feller, U. Drought stress effects on Rubisco in wheat: Changes in the Rubisco large subunit. Acta Physiol. Plant. 2009, 31, 1129-1138. [CrossRef]

16. Farooq, M.; Rizwan, M.; Nawaz, A.; Rehman, A.; Ahmad, R. Application of natural plant extracts improves the tolerance against combined terminal heat and drought stresses in bread wheat. J. Agron. Crop Sci. 2017, 203, 528-538. [CrossRef]

17. Desoky, E.M.; Merwad, A.M.A.; Rady, M.M. Natural biostimulants improve saline soil characteristics and salt stressed-sorghum performance. Commun. Soil Sci. Plant Anal. 2018, 49, 967-983. [CrossRef]

18. Sangiorgio, D.; Cellini, A.; Donati, I.; Pastore, C.; Onofrietti, C.; Spinelli, F. Facing climate change: Application of microbial biostimulants to mitigate stress in horticultural crops-A review. Agronomy 2020, 10, 794. [CrossRef]

19. Silva, E.O.; Martins, S.J.; Alves, E. Essential oils for the control of bacterial speck in tomato crop. Afr. J. Agric. Res. 2014, 9, 2624-2629. [CrossRef]

20. Martins, S.J.; Medeiros, F.H.V.; Lakshmanan, V.; Bais, H.P. Impact of seed exudates on growth and biofilm formation of Bacillus amyloliquefaciens ALB629 in common bean. Front. Microbiol. 2018, 8, 2631. [CrossRef]

21. Martins, S.J.; Rocha, G.A.; Georg, R.C.; Ulhôa, C.J.; Cunha, M.G.; Rocha, M.R.; Araújo, L.G.; Vaz, K.S.; Dianese, E.C.; Oshiquiri, L.H.; et al. Plant-associated bacteria mitigate drought stress in soybean. Environ. Sci. Pollut. Res. 2018, 25, 13676-13686. [CrossRef]

22. Semida, W.M.; Rady, M.M. Presoaking application of propolis and maize grain extracts alleviates salinity stress in common bean (Phaseolus vulgaris L.). Sci. Hortic. 2014, 168, 210-217. [CrossRef]

23. Rady, M.M.; Kuşvuran, A.; Alharby, H.F.; Alzahrani, Y.; Kuşvuran, S. Pretreatment with proline or an organic bio-stimulant induces salt tolerance in wheat plants by improving antioxidant redox state and enzymatic activities and reducing the oxidative stress. J. Plant Growth Regul. 2019, 38, 449-462. [CrossRef]

24. Rehman, H.; Alharby, H.F.; Alzahrani, Y.; Rady, M.M. Magnesium and organic biostimulant integrative application induces physiological and biochemical changes in sunflower plants and its harvested progeny on sandy soil. Plant Physiol. Biochem. 2018, 126, 97-105. [CrossRef] [PubMed] 
25. Alzahrani, Y.; Rady, M.M. Compared to antioxidants and polyamines, the role of maize grain-derived organic biostimulants in improving cadmium tolerance in wheat plants. Ecotoxicol. Environ. Saf. 2019, 182, 109378. [CrossRef]

26. Alharby, H.F.; Alzahrani, Y.M.; Rady, M.M. Seeds pretreatment with zeatins or maize grain-derived organic biostimulant improved hormonal contents, polyamine gene expression, and salinity and drought tolerance of wheat. Int. J. Agric. Biol. 2020, 24, 714-724.

27. Rady, M.M.; Talaat, N.; Abdelhamid, M.T.; Shawky, B.T.; Desoky, E.M. Maize (Zea mays L.) grains extract mitigates the deleterious effects of salt stress on common bean (Phaseolus vulgaris L.) growth and physiology. J. Hortic. Sci. Biotechnol. 2019, 94, 777-789. [CrossRef]

28. Denby, K.; Gehring, C. Engineering drought and salinity tolerance in plants: Lessons from genome-wide expression profiling in Arabidopsis. Trends Biotechnol. 2005, 23, 547-552. [CrossRef]

29. Rady, M.M.; Hemida, K.A. Modulation of cadmium toxicity and enhancing cadmium-tolerance in wheat seedlings by exogenous application of polyamines. Ecotoxicol. Environ. Saf. 2015, 119, 178-185. [CrossRef]

30. Sundstrom, F.J.; Reader, R.B.; Edwards, R.L. Effect of seed treatment and planting method on Tabasco pepper. J. Amer. Soc. Hortic. Sci. 1987, 112, 641-644.

31. Hoagland, D.R.; Arnon, D.I. The Water Culture Method for Growing Plants without Soil; College of Agriculture, Agricultural Experiment Station, University of California: Baltimore, MA, USA, 1950.

32. Kampfenkel, K.; Van Montagu, M. Extraction and determination of ascorbate and dehydroascorbate from plant tissue. Anal. Biochem. 1995, 225, 165-167. [CrossRef] [PubMed]

33. Griffith, O.W. Determination of glutathione and glutathione disulfide using glutathione reductase and 2-vinylpyridine. Anal. Biochem. 1980, 106, 207-212. [CrossRef]

34. Lee, S.C.; Kim, J.H.; Jeong, S.M.; Kim, D.R.; Ha, J.U.; Nam, K.C. Effect of far-infrared radiation on the antioxidant activity of rice hulls. J. Agric. Food Chem. 2003, 51, 4400-4403. [CrossRef]

35. Lavrich, R.J.; Hays, M.D. Validation studies of thermal extraction-GC/MS applied to source emissions aerosols. 1. Semivolatile analyte-nonvolatile matrix interactions. Anal. Chem. 2007, 79, 3635-3645. [CrossRef]

36. Chen, J.; Guo, Z.; Fang, J.; Lu, S. Physiological responses of a centipedegrass mutant to chilling stress. Agron. J. 2013, 105, 1814-1820. [CrossRef]

37. Guo, Z.; Tan, J.; Zhuo, C.; Wang, C.; Xiang, B.; Wang, Z. Abscisic acid, $\mathrm{H}_{2} \mathrm{O}_{2}$ and nitric oxide interactions mediated cold-induced S-adenosylmethionine synthetase in Medicago sativa subsp. falcata that confers cold tolerance through up-regulating polyamine oxidation. Plant Biotechnol. J. 2014, 12, 601-612. [CrossRef]

38. Flores, E.; Galston, A.W. Analysis of polyamines in higher plants by high performance liquid chromatography. Plant Physiol. 1982, 69, 701-706. [CrossRef]

39. Arnon, D.I. Copper enzymes in isolated chloroplasts, polyphenoxidase in Beta vulgaris. Plant Physiol. 1949, 24, 1-15. [CrossRef]

40. Maxwell, K.; Johnson, G.N. Chlorophyll fluorescence-A practical guide. J. Exp. Bot. 2000, 51, 659-668. [CrossRef]

41. Clark, A.J.; Landolt, W.; Bucher, J.B.; Strasser, R.J. Beech (Fagus sylvatica) response to ozone exposure assessed with a chlorophyll a fluorescence performance index. Environ. Pollut. 2000, 109, 501-507. [CrossRef]

42. Bregman, A. Laboratory Investigations in Cell and Molecular Biology, 3rd ed.; John Wiley \& Sons: New York, NY, USA, 1990.

43. Velikova, V.; Yordanov, I.; Edreva, A. Oxidative stress and some antioxidant systems in acid rain treated bean plants. Plant Sci. 2000, 151, 59-66. [CrossRef]

44. Kubis, J. Exogenous spermidine differentially alters activities of some scavenging system enzymes, $\mathrm{H}_{2} \mathrm{O}_{2}$ and superoxide radical levels in water-stressed cucumber leaves. J. Plant Physiol. 2008, 165, 397-406. [CrossRef]

45. Madhava Rao, K.V.; Sresty, T.V.S. Antioxidative parameters in the seedlings of pigeonpea (Cajanus cajan (L.) Millspaugh) in response to Zn and Ni stresses. Plant Sci. 2000, 157, 113-128. [CrossRef]

46. Rady, M.M. Effect of 24-epibrassinolide on growth, yield, antioxidant system and cadmium content of bean (Phaseolus vulgaris L.) plants under salinity and cadmium stress. Sci. Hortic. 2011, 129, 232-237. [CrossRef]

47. Osman, A.S.; Rady, M.M. Effect of humic acid as an additive to growing media to enhance the production of eggplant and tomato transplants. J. Hortic. Sci. Biotechnol. 2014, 89, 237-244. [CrossRef]

48. Dhindsa, R.S.; Matowe, W. Drought tolerance in two mosses: Correlated with enzymatic defence against lipid peroxidation. J. Exp. Bot. 1981, 32, 79-91. [CrossRef]

49. Aebi, H. Catalase in vitro. In Methods in Enzymology; Elsevier: Amsterdam, The Netherlands, 1984; pp. $121-126$.

50. Hasanuzzaman, M.; Fujita, M. Exogenous sodium nitroprusside alleviates arsenic-induced oxidative stress in wheat (Triticum aestivum L.) seedlings by enhancing antioxidant defense and glyoxalase system. Ecotoxicology 2013, 22, 584-596. [CrossRef]

51. Nakano, Y.; Asada, K. Hydrogen peroxide is scavenged by ascorbate-specific peroxidase in spinach chloroplasts. Plant Cell Physiol. 1981, 22, 867-880.

52. Foster, J.G.; Hess, J.L. Responses of superoxide dismutase and glutathione reductase activities in cotton leaf tissue exposed to an atmosphere enriched in oxygen. Plant Physiol. 1980, 66, 482-487. [CrossRef]

53. Miyake, C.; Asada, K. Thylakoid-bound ascorbate peroxidase in spinach chloroplasts and photoreduction of its primary oxidation product monodehydroascorbate radicals in thylakoids. Plant Cell Physiol. 1992, 33, 541-553.

54. Huang, C.; He, W.; Guo, J.; Chang, X.; Su, P.; Zhang, L. Increased sensitivity to salt stress in an ascorbate-deficient Arabidopsis mutant. J. Exp. Bot. 2005, 56, 3041-3049. [CrossRef] 
55. Yu, C.W.; Murphy, T.M.; Lin, C.H. Hydrogen peroxide-induced chilling tolerance in mung beans mediated through ABAindependent glutathione accumulation. Funct. Plant Biol. 2003, 30, 955. [CrossRef] [PubMed]

56. Paradiso, A.; Berardino, R.; de Pinto, M.C.; di Toppi, L.S.; Storelli, M.M.; Tommasi, F.; De Gara, L. Increase in ascorbate-glutathione metabolism as local and precocious systemic responses induced by cadmium in durum wheat plants. Plant Cell Physiol. 2008, 49, 362-374. [CrossRef] [PubMed]

57. Bates, L.S.; Waldren, R.P.; Teare, I.D. Rapid determination of free proline for water-stress studies. Plant Soil 1973, 39, $205-207$. [CrossRef]

58. Grieve, C.M.; Grattan, S.R. Rapid assay for determination of water soluble quaternary ammonium compounds. Plant Soil 1983, 70, 303-307. [CrossRef]

59. Irigoyen, J.J.; Einerich, D.W.; Sánchez-Díaz, M. Water stress induced changes in concentrations of proline and total soluble sugars in nodulated alfalfa (Medicago sativa) plants. Physiol. Plant. 1992, 84, 55-60. [CrossRef]

60. Novák, O.; Hauserová, E.; Amakorová, P.; Doležal, K.; Strnad, M. Cytokinin profiling in plant tissues using ultra-performance liquid chromatography-electrospray tandem mass spectrometry. Phytochemistry 2008, 69, 2214-2224. [CrossRef]

61. Forcat, S.; Bennett, M.H.; Mansfield, J.W.; Grant, M.R. A rapid and robust method for simultaneously measuring changes in the phytohormones ABA, JA and SA in plants following biotic and abiotic stress. Plant Methods 2008, 4, 16. [CrossRef]

62. Gomez, K.A.; Gomez, A.A. Statistical Procedures for Agricultural Research, 2nd ed.; John Wiley \& Sons: Singapore, $1984 ;$ p. 680.

63. Taha, R.S.; Alharby, H.F.; Bamagoos, A.A.; Medani, R.A.; Rady, M.M. Elevating tolerance of drought stress in Ocimum basilicum using pollen grains extract; a natural biostimulant by regulation of plant performance and antioxidant defense system. S. Afr. J. Bot. 2020, 128, 42-53. [CrossRef]

64. Noctor, G.; Veljovic-Jovanovic, S.; Driscoll, S.; Novitskaya, L.; Foyer, C.H. Drought and oxidative load in the leaves of $C_{3}$ plants: A predominant role for photorespiration? Ann. Bot. 2002, 89, 841-850. [CrossRef]

65. Wang, H.-Z.; Zhang, L.-H.; Ma, J.; Li, X.-Y.; Li, Y.; Zhang, R.-P.; Wang, R.-Q. Effects of water stress on reactive oxygen species generation and protection system in rice during grain-filling stage. Agric. Sci. China 2010, 9, 633-641. [CrossRef]

66. Gill, S.S.; Tuteja, N. Polyamines and abiotic stress tolerance in plants. Plant Signal. Behav. 2010, 5, 26-33. [CrossRef] [PubMed]

67. Ebeed, H.T.; Hassan, N.M.; Aljarani, A.M. Exogenous applications of Polyamines modulate drought responses in wheat through osmolytes accumulation, increasing free polyamine levels and regulation of polyamine biosynthetic genes. Plant Physiol. Biochem. 2017, 118, 438-448. [CrossRef]

68. Taie, H.A.A.; El-Yazal, M.A.S.; Ahmed, S.M.A.; Rady, M.M. Polyamines modulate growth, antioxidant activity, and genomic DNA in heavy metal-stressed wheat plant. Environ. Sci. Pollut. Res. 2019, 26, 22338-22350. [CrossRef]

69. Yildiztugay, A.; Ozfidan-Konakci, C.; Yildiztugay, E.; Kucukoduk, M. Biochar triggers systemic tolerance against cobalt stress in wheat leaves through regulation of water status and antioxidant metabolism. J. Soil Sci. Plant Nutr. 2019, 19, 935-947. [CrossRef]

70. Ghassemi, S.; Farhangi-Abriz, S.; Faegi-Analou, R.; Ghorbanpour, M.; Lajayer, B.A. Monitoring cell energy, physiological functions and grain yield in field-grown mung bean exposed to exogenously applied polyamines under drought stress. J. Soil Sci. Plant Nutr. 2018, 18, 1108-1125. [CrossRef]

71. Dalal, V.K.; Tripathy, B.C. Modulation of chlorophyll biosynthesis by water stress in rice seedlings during chloroplast biogenesis. Plant Cell Environ. 2012, 35, 1685-1703. [CrossRef]

72. Zaki, S.S.; Rady, M.M. Moringa oleifera leaf extract improves growth, physio-chemical attributes, antioxidant defence system and yields of salt-stressed Phaseolus vulgaris L. plants. Int. J. Chem. Tech. Res. 2015, 8, 120-134.

73. Cortleven, A.; Marg, I.; Yamburenko, M.V.; Schlicke, H.; Hill, K.; Grimm, B.; Schaller, G.E.; Schmülling, T. Cytokinin regulates the etioplast-chloroplast transition through the two-component signaling system and activation of chloroplast-related genes. Plant Physiol. 2016, 172, 464-478. [CrossRef]

74. Tikkanen, M.; Rantala, S.; Aro, E.-M. Electron flow from PSII to PSI under high light is controlled by PGR5 but not by PSBS. Front. Plant Sci. 2015, 6, 521. [CrossRef]

75. Behera, R.K.; Mishra, P.C.; Choudhury, N.K. High irradiance and water stress induce alterations in pigment composition and chloroplast activities of primary wheat leaves. J. Plant Physiol. 2002, 159, 967-973. [CrossRef]

76. Aldesuquy, H. Synergistic effect of phytohormones on pigment and fine structure of chloroplasts in flag leaf of wheat plants irrigated by seawater. Egypt. J. Basic Appl. Sci. 2015, 2, 310-317. [CrossRef]

77. Singh, S.; Prasad, S.M. Growth, photosynthesis and oxidative responses of Solanum melongena L. seedlings to cadmium stress: Mechanism of toxicity amelioration by kinetin. Sci. Hortic. 2014, 176, 1-10. [CrossRef]

78. Mittler, R. Oxidative stress, antioxidants and stress tolerance. Trends Plant Sci. 2002, 7, 405-410. [CrossRef]

79. Ahanger, M.A.; Alyemeni, M.N.; Wijaya, L.; Alamri, S.A.; Alam, P.; Ashraf, M.; Ahmad, P. Potential of exogenously sourced kinetin in protecting Solanum lycopersicum from NaCl-induced oxidative stress through up-regulation of the antioxidant system, ascorbate-glutathione cycle and glyoxalase system. PLOS ONE 2018, 13, e0202175. [CrossRef]

80. Foyer, C.H.; Noctor, G. Ascorbate and glutathione: The heart of the redox hub. Plant Physiol. 2011, 155, 2-18. [CrossRef]

81. Wu, X.; Zhu, Z.; Li, X.; Zha, D. Effects of cytokinin on photosynthetic gas exchange, chlorophyll fluorescence parameters and antioxidative system in seedlings of eggplant (Solanum melongena L.) under salinity stress. Acta Physiol. Plant. 2012, 34, $2105-2114$. [CrossRef] 
82. Gupta, B.K.; Sahoo, K.K.; Ghosh, A.; Tripathi, A.K.; Anwar, K.; Das, P.; Singh, A.K.; Pareek, A.; Sopory, S.K.; Singla-Pareek, S.L. Manipulation of glyoxalase pathway confers tolerance to multiple stresses in rice. Plant Cell Environ. 2017, 41, 1186-1200. [CrossRef]

83. Li, Z.G. Methylglyoxal and glyoxalase system in plants: Old players, new concepts. Bot. Rev. 2016, 82, 183-203. [CrossRef]

84. Yadav, S.K.; Singla-Pareek, S.L.; Reddy, M.; Sopory, S.K. Methylglyoxal detoxification by glyoxalase system: A survival strategy during environmental stresses. Physiol. Mol. Biol. Plant 2005, 11, 1.

85. Ahanger, M.A.; Agarwal, R.M. Salinity stress induced alterations in antioxidant metabolism and nitrogen assimilation in wheat (Triticum aestivum L) as influenced by potassium supplementation. Plant Physiol. Biochem. 2017, 115, 449-460. [CrossRef]

86. Delauney, A.J.; Verma, D.P.S. Proline biosynthesis and osmoregulation in plants. Plant J. 1993, 4, 215-223. [CrossRef]

87. Ahmad, P. Growth and antioxidant responses in mustard (Brassica juncea L.) plants subjected to combined effect of gibberellic acid and salinity. Arch. Agron. Soil Sci. 2010, 56, 575-588. [CrossRef]

88. Thakur, M.; Sharma, A.D. Salt-stress-induced proline accumulation in germinating embryos: Evidence suggesting a role of proline in seed germination. J. Arid Environ. 2005, 62, 517-523. [CrossRef]

89. Hai, N.N.; Chuong, N.N.; Tu, N.H.C.; Kisiala, A.; Hoang, X.L.T.; Thao, N.P. Role and Regulation of Cytokinins in Plant Response to Drought Stress-A review. Plants 2020, 9, 422. [CrossRef]

90. Gajdosová, S.; Spichal, L.; Kaminek, M.; Hoyerová, K.; Novák, O.; Dobrev, P.I.; Galuszka, P.; Klıma, P.; Gaudinova, A.; Zizkova, E.; et al. Distribution, biological activities, metabolism, and the conceivable function of cis-zeatin-type cytokinins in plants. J. Exp. Bot. 2011, 62, 2827-2840. [CrossRef]

91. Kudo, T.; Makita, N.; Kojima, M.; Tokunaga, H.; Sakakibara, H. Cytokinin activity of cis-zeatin and phenotypic alterations induced by overexpression of putative cis-Zeatin-O-glucosyltransferase in rice. Plant Physiol. 2012, 160, 319-331. [CrossRef] 